\title{
Graph complexes in deformation quantization
}

\author{
Domenico Fiorenza and Lucian M. Ionescu
}

October 22, 2018

\begin{abstract}
Kontsevich's formality theorem and the consequent star-product formula rely on the construction of an $L_{\infty}$-morphism between the DGLA of polyvector fields and the DGLA of polydifferential operators. This construction uses a version of graphical calculus. In this article we present the details of this graphical calculus with emphasis on its algebraic features. It is a morphism of differential graded Lie algebras between the Kontsevich DGLA of admissible graphs and the Chevalley-Eilenberg DGLA of linear homomorphisms between polyvector fields and polydifferential operators.

Kontsevich's proof of the formality morphism is reexamined in this light and an algebraic framework for discussing the tree-level reduction of Kontsevich's star-product is described.
\end{abstract}

\section{Contents}

1 Introduction 2

2 The graphical calculus for derivations 3

2.1 Kontsevich's admissible graphs . . . . . . . . . . . . . . 3

2.2 Graphical calculus for derivations . . . . . . . . . . . . . 4

2.3 A computation example . . . . . . . . . . . . . . 6

$3 \quad$ The Chevallev-Eilenberg DGLA $C E^{\bullet}\left(T^{\bullet} D^{\bullet}, 7\right.$

3.1 The Lie algebra $T^{\bullet} \ldots \ldots \ldots \ldots \ldots$. . . . . . . . . . . 7

3.2 The Lie algebra $D^{\bullet} \ldots \ldots \ldots \ldots \ldots$

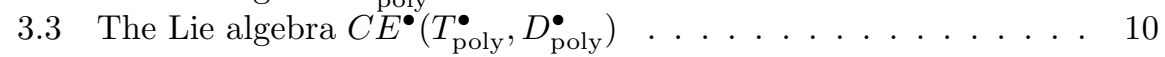

4 The Maurer-Cartan equation and $L_{\infty}$-morphisms 12

4.1 The Maurer-Cartan equation on the Chevallev-Eilenberg complex 12

4.2 The quantization of Poisson structures . . . . . . . . . . . . 14

4.3 The vertical Maurer-Cartan equation . . . . . . . . . . . . . . 14

5 The differential graded Lie algebra of graphs $\quad 15$

5.1 The o composition of graphs . . . . . . . . . . . . . . 17

5.2 The differential $d_{0}$ on graphs . . . . . . . . . . . . . . . 18

5.3 The total differential on graphs . . . . . . . . . . . . . . . . . 19 
6 The map $\mathcal{U}$ as a morphism of differential graded Lie algebras 20

$6.1 \mathcal{U}$ as a morphism of Lie algebras . . . . . . . . . . . . . . 20

$6.2 \mathcal{U}$ as a morphism of complexes . . . . . . . . . . . . . 22

7 The Maurer-Cartan equation on graphs and the cocvcle equation 24

7.1 The dual dg-Lie-coalgebra structure . . . . . . . . . . . 24

7.2 The Maurer-Cartan equation on graphs and the cocvcle equation 25

7.3 Connes-Kreimer convolution and Kontsevich solution . . . . . . . 28

7.4 Configuration spaces and the cobar construction . . . . . . . 28

8 The tree-level approximation $\quad 29$

A Appendix: Configuration spaces and Kontsevich's cocvcle 32

A.1 Configuration spaces and differential forms associated with graphs 32

A.2 Normal subgraphs and the boundarv behavior of the $\omega_{\Gamma}$ 's . . . . 33

A.3 Stokes' theorem and the cocvcle equation . . . . . . . . . . 36

\section{Introduction}

In the breakthrough paper Kon97 solving the long-standing problem of deformation quantization, Maxim Kontsevich makes use of a perturbative approach in writing his solution: the formality $L_{\infty}$-quasi-isomorphism. The ideological background is that of a string theory and, as it was explained in CF99, the formality morphism is really the perturbative expansion of the partition function of a sigma model on the unit disk with the 2-point function yielding the starproduct. From a mathematical point of view, Kontsevich's construction can be seen as a graphical calculus for derivations, providing another example of the ubiquity of graphical calculi in contemporary mathematics (e.g. Feynman diagrams, Turaev's tangles, knot and 3-manifold invariants, TQFTs, operads and PROPs etc.). The analogy between Kontsevich's and Feynman's graphical calculus was further pointed out in [on03.

In this article we focus on the graphical calculus for derivations from the mathematical point of view, insisting on the fact that the Kontsevich's rule implements graphically an action, which extends the basic rule associating to a colored arrow the corresponding evaluation

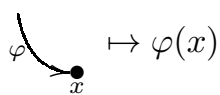

by incorporating the Leibniz rule relative to the natural tensor product given by disjoint union:

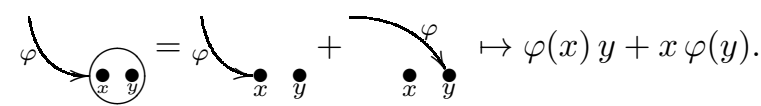

The main result is Theorem 6.1 stating that Kontsevich's graphical calculus is a DGLA morphism between the differential graded Lie algebra $\mathscr{G} \bullet$ of Kontsevich's admissible graphs and the Chevalley-Eilenberg differential graded Lie 
algebra $C E^{\bullet}\left(T_{\text {poly }}^{\bullet}, D_{\text {poly }}^{\bullet}\right)$ of linear homomorphisms between polyvector fields and polydifferential operators. From this it follows that a solution of the MaurerCartan equation in $\mathscr{G} \bullet$ induces by push-forward a solution of the Maurer-Cartan equation in $C E^{\bullet}\left(T_{\text {poly }}^{\bullet}, D_{\text {poly }}^{\bullet}\right)$; therefore it provides an $L_{\infty}$-morphism between $T_{\text {poly }}^{\bullet}$ and $D_{\text {poly }}^{\bullet}$, which in turn can be used to define a star-product quantization of Poisson structures. The interplay between the DGLA structure on admissible graphs and its dual dg-Lie-coalgebra structure is mentioned, pointing out its rôle in reducing the problem of solving the Maurer-Cartan equation on graphs to the problem of finding a system of "weights" $W_{\Gamma}$ satisfying the cocycle equation which is dual to the Maurer-Cartan equation.

In the final part of the paper, we show how the DGLA structure on $\mathscr{G} \bullet$ provides a nice algebraic framework for studying the tree-level approximation of Kontsevich's star product formula. Indeed, by homotopical transfer of structure, the DGLA structure on $\mathscr{G} \bullet$ induces an $L_{\infty}$-algebra structure on the subcomplex $\mathscr{F} \bullet$ of forest graphs, and an $L_{\infty}$-morphism $\mathcal{U}_{\infty}: \mathscr{F} \bullet \rightarrow C E^{\bullet}\left(T_{\text {poly }}^{\bullet}, D_{\text {poly }}^{\bullet}\right)$, whose principal part is the Kontsevich's graphical calculus restricted to forest graphs. As a consequence one obtains that it is possible to write a star-product formula as a sum over forest graphs; this formula is given by a Kontsevich-type formula involving only forest graphs (the so-called tree-level or semi-classical approximation) plus non-linear corrections corresponding to non simply-connected graphs in the original Kontsevich's formula.

There is a considerable overlap between the first few sections of this paper and other expositions of Kontsevich's paper Cat04, Kel, DS02, AMM02] etc., but we tried to reorganize the presentation to clarify the DGLA structure on graphs, together with the key feature of Kontsevich's approach, namely that the graphical calculus $\mathcal{U}$ is a DGLA morphism.

Acknowledgments. The authors thank Benoit Dherin, Fabio Gavarini and the Referees from Letters in Mathematical Physics for very useful comments and suggestions on the first version of this paper.

\section{The graphical calculus for derivations}

An oriented graph can be used to build a differential operator out of a finite set of polyvector fields. This construction is quite standard (see, for instance Kon97) and we will recall only its computational rules here. To begin with, we need to specify which graphs we do have in mind.

\subsection{Kontsevich's admissible graphs}

By definition, an admissible graph with $n$ internal vertices and $m$ boundary vertices is a finite oriented graph $\Gamma$ endowed with the following additional data/ conditions:

1. the vertices of $\Gamma$ are split into the two totally ordered disjoint subsets of internal and boundary vertices; 
2. the internal vertices are numbered from 1 to $n$;

3. the boundary vertices are numbered from 1 to $m$;

4. no edge stems from a boundary vertex, i.e., boundary vertices are sinks;

5. an ordering on the edges stemming from each internal vertex is given; due to the total order on the internal vertices, this is equivalent to a total order on the set of all edges of $\Gamma$;

6. there are no multiple edges, and no loops, i.e. edges starting and ending on the same vertex.

A good way of visualizing admissible graphs is as graphs drawn into a disk, with internal vertices in the interior of the disk and boundary vertices on the boundary, in such a way that all their edges are realized as geodesics for the standard hyperbolic metric of the disk. An example is given in the following figure, depicting an admissible graph with 2 internal vertices and 3 boundary vertices:

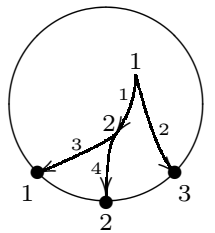

Figure 1: an admissible graph

\subsection{Graphical calculus for derivations}

Let now $x^{i}$ be coordinates on $\mathbb{R}^{d}$, and let $\partial_{i}$ be the corresponding vector fields. The space of polyvector fields on $\mathbb{R}^{d}$ is

$$
T_{\text {poly }}^{\bullet}=\bigoplus_{k=-1}^{\infty} T_{\text {poly }}^{k}=\bigoplus_{k=-1}^{\infty} H^{0}\left(\mathbb{R}^{d} ; \wedge^{k+1} T \mathbb{R}^{d}\right)
$$

The graphical calculus for derivations uses a graph $\Gamma$ with $n$ internal and $m$ boundary vertices, together with $n$ polyvector fields, to build a polydifferential operator acting on $m$ smooth functions defined on $\mathbb{R}^{d}$. In other words, if we denote by $D_{\text {poly }}^{m-1}$ the space of polydifferential operators acting on $m$ smooth functions defined on $\mathbb{R}^{d}$ (3.2 see also Cat04, p.24), then the graphical calculus for derivations associates to a graph $\Gamma$ with $n$ internal and $m$ boundary vertices a map

$$
\mathcal{U}_{\Gamma}:\left(T_{\text {poly }}^{\bullet}\right)^{\otimes n} \rightarrow D_{\text {poly }}^{m-1} .
$$

More precisely, if $\nu_{r}+1$ is the number of edges stemming from the $r^{\text {th }}$ internal vertex of $\Gamma$, then $\mathcal{U}_{\Gamma}$ is a map

$$
\mathcal{U}_{\Gamma}: \bigotimes_{r=1}^{n} T_{\text {poly }}^{\nu_{r}} \rightarrow D_{\text {poly }}^{m-1}
$$


which is better expressed in the grading preserving form

$$
\mathcal{U}_{\Gamma}: \bigotimes_{r=1}^{n} T_{\text {poly }}^{\nu_{r}} \rightarrow\left(D_{\text {poly }}[v(\Gamma)-1-e(\Gamma)]\right)^{\sum \nu_{r}}
$$

where $e(\Gamma)$ and $v(\Gamma)$ are the number of edges and vertices of $\Gamma$, respectively, and we have used the identities

$$
\begin{aligned}
& e(\Gamma)=n+\sum_{r=1}^{n} \nu_{r} ; \\
& v(\Gamma)=m+n .
\end{aligned}
$$

This suggests to introduce a bigrading on admissible graphs as follows.

Definition 2.1. An admissible graph of type $(p, q)$ is an admissible graph $\Gamma$ such that

1. $p=\chi(\Gamma)-1=v(\Gamma)-e(\Gamma)-1$;

2. the number of internal vertices of $\Gamma$ is $q$.

The set of isomorphism classes ${ }^{1}$ of admissible graphs of type $(p, q)$ will be denoted by the symbol $\mathcal{G}^{p, q}$.

With these notations, $\mathcal{U}$ can be seen as a map

$$
\mathcal{U}: \mathcal{G}^{p, q} \rightarrow \operatorname{Hom}\left(\left(T_{\text {poly }}^{\bullet}\right)^{\otimes q}, D_{\text {poly }}^{\bullet}[p]\right),
$$

where the homomorphisms on the right hand side are grading preserving. We will return on this in the following sections.

Now, let us explicitly describe the rules of graphical calculus. Such rules define

$$
\left\langle\mathcal{U}_{\Gamma}\left(\xi_{1} \otimes \cdots \otimes \xi_{n}\right) \mid f_{0} \otimes \cdots \otimes f_{m-1}\right\rangle
$$

as a state sum. Namely, evaluation of the polydifferential operator above is computed by considering the decorated graph corresponding to our data, i.e., the graph $\Gamma$ with the internal vertices labeled by the polyvector fields $\left\{\xi_{r}\right\}_{r=1, \ldots, n}$ and the boundary vertices labeled by the functions $\left\{f_{j}\right\}_{j=1, \ldots, m}$. Now proceed to a state-sum construction; the edges stemming from each internal vertex $v$ of $\Gamma$ are labeled by tensor indices $i_{k}$ with $k$ given by the numbering induced by the total order on the set of edges. We refer to this operation as "coloring" the graph.

The value of such a colored graph is defined as the product over the contributions of the vertices of $\Gamma$, where the value of a decorated vertex $v$ is

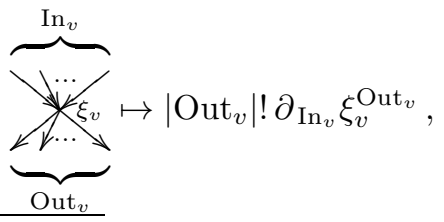

\footnotetext{
${ }^{1} \mathrm{An}$ isomorphism of admissible graphs is an isomorphism of the underlying oriented graphs which preserves all the additional structures on them.
} 
for an internal vertex, and

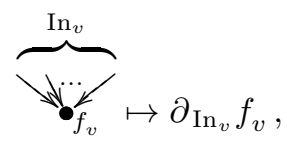

for a boundary vertex, where $\operatorname{In}_{v}$ and Out ${ }_{v}$ are the multi-indices corresponding to the numbering on the incoming and outgoing edges at the vertex $v$. Finally, we sum over all repeated indices according to Einstein's convention.

Remark 2.1. Polydifferential operators can be built by two basic operations: multiplication of functions, and $m$-vector fields acting as polyderivations on $m$ functions. These two basic operations are represented in the graphical calculus for derivations $\mathcal{U}$ by the most basic graphs. Namely, multiplication of $m$ functions is represented by the graph $b_{0, m}$ with no internal vertices and $m$ boundary vertices:

$$
\left\langle\mathcal{U}_{b_{0, m}} \mid f_{0} \otimes f_{1} \otimes \cdots \otimes f_{m-1}\right\rangle=\left(f_{f_{0} \cdots} \cdots f_{f_{1}} \cdots f_{m-1}\right.
$$

It is an element of $\mathcal{G}^{m-1,0}$. The identification of $m$-vector fields with polyderivations is represented by the admissible graph $b_{1, m}$ with one internal vertex, $m$ boundary vertices and $m$ edges (we will call $b_{1, m}$ an $m$-corolla):

$$
\begin{gathered}
\left\langle\mathcal{U}_{b_{1, m}}(\xi) \mid f_{0} \otimes f_{1} \otimes \cdots \otimes f_{m-1}\right\rangle= \\
=m ! \xi^{i_{0} i_{1} \ldots i_{m-1}}\left(\partial_{i_{0}} f_{0}\right)\left(\partial_{i_{1}} f_{1}\right) \cdots\left(\partial_{i_{m-1}} f_{m-1}\right) .
\end{gathered}
$$

It is an element of $\mathcal{G}^{0,1}$, independently of $m$.

\subsection{A computation example}

We want to compute the pairing

$$
\left\langle\mathcal{U}_{\Gamma}\left(\xi_{1} \otimes \xi_{2}\right) \mid f \otimes g \otimes h\right\rangle,
$$

where $\Gamma$ is the graph of Figure 1 in $\$ 2.1$ The first step consists in decorating the boundary vertices with the functions $f, g$ and $h$, and the internal vertices 
with the polyvector fields $\xi_{1}$ and $\xi_{2}$ :

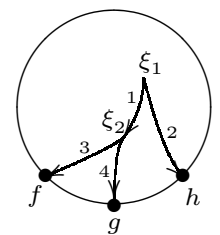

Note that both the internal vertices of $\Gamma$ have two outgoing edges. This means that both $\xi_{1}$ and $\xi_{2}$ have to be bi-vector fields in order to have a non-trivial pairing. Let us write $\xi_{1}=\xi_{1}^{i_{1} i_{2}} \partial_{i_{1}} \wedge \partial_{i_{2}}$ and $\xi_{2}=\xi_{2}^{i_{3} i_{4}} \partial_{i_{3}} \wedge \partial_{i_{4}}$. We now "color" the edges of $\Gamma$. The $k^{\text {th }}$ edge is labeled by $i_{k}$ :

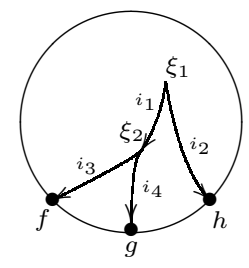

Now we are left with the task of giving a value to the above colored graph. To do this we look at the values of the vertices:

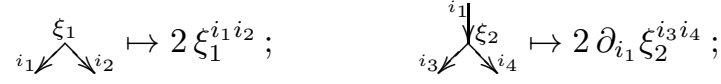

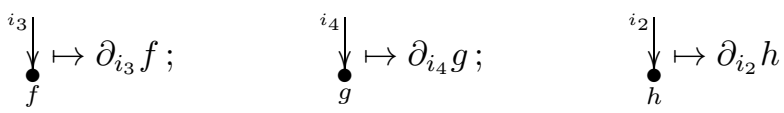

and multiply them. In conclusion, we get the formula

$$
\left\langle\mathcal{U}_{\Gamma}\left(\xi_{1} \otimes \xi_{2}\right) \mid f \otimes g \otimes h\right\rangle=4 \xi_{1}^{i_{1} i_{2}}\left(\partial_{i_{1}} \xi_{2}^{i_{3} i_{4}}\right)\left(\partial_{i_{3}} f\right)\left(\partial_{i_{4}} g\right)\left(\partial_{i_{2}} h\right) .
$$

\section{The Chevalley-Eilenberg DGLA $C E^{\bullet}\left(T_{\text {poly }}^{\bullet}, D_{\text {poly }}^{\bullet}\right)$}

We review the Lie algebra structures of the main DGLAs involved, together with the corresponding canonical differentials. This is standard material included for the sake of completeness.

\subsection{The Lie algebra $T_{\text {poly }}^{\bullet}$}

The space $T_{\text {poly }}^{\bullet}$ of polyvector fields on $\mathbb{R}^{d}$ is endowed with a graded Lie algebra structure by the Schouten-Nijenhuis bracket:

$$
\begin{aligned}
& {\left[\xi_{0} \wedge \cdots \wedge \xi_{k}, \eta_{0} \wedge \cdots \wedge \eta_{l}\right]_{S N}=} \\
& \quad=\sum_{i, j}(-1)^{i+j+k}\left[\xi_{i}, \eta_{j}\right] \wedge \xi_{0} \wedge \cdots \wedge \hat{\xi}_{i} \wedge \cdots \wedge \xi_{k} \wedge \eta_{0} \wedge \cdots \wedge \hat{\eta}_{j} \wedge \cdots \wedge \eta_{l},
\end{aligned}
$$


where $k, l \geq 0$ and $\xi_{i}, \eta_{j} \in T_{\text {poly }}^{0}$; the hat over a factor denotes its absence. We will use the shorthand notation

$$
\xi_{\bar{i}}:=\xi_{o} \wedge \cdots \wedge \hat{\xi}_{i} \wedge \cdots \wedge \xi_{k}
$$

so that the Schouten-Nijenhuis bracket is written as

$$
\left[\xi_{0} \wedge \cdots \wedge \xi_{k}, \eta_{0} \wedge \cdots \wedge \eta_{l}\right]_{S N}=\sum_{i=0}^{k} \sum_{j=0}^{l}(-1)^{i+j+k}\left[\xi_{i}, \eta_{j}\right] \wedge \xi_{\bar{i}} \wedge \eta_{\bar{j}}
$$

The Schouten-Nijenhuis bracket is induced by a pre-Lie operation on polyvector fields, the Nijenhuis-Richardson pre-Lie multiplication (Kon97, p.15; Kel], Moc02 p.81). By this we mean that there is a (non associative) multiplication

$$
\text { •: } T_{\text {poly }}^{k} \otimes T_{\text {poly }}^{l} \rightarrow T_{\text {poly }}^{k+l}
$$

such that ([Kon97, p.15):

$$
[\xi, \eta]_{S N}=\xi \bullet \eta-(-1)^{k l} \eta \bullet \xi .
$$

The definition of the Nijenhuis-Richardson $\bullet$ operation is the following: if $\xi=$ $\xi_{0} \wedge \cdots \wedge \xi_{k}$ and $\eta=\eta_{0} \wedge \cdots \wedge \eta_{l}$, with $\xi_{i}, \eta_{j} \in T_{\text {poly }}^{0}$, then

$$
\xi \bullet \eta=\sum_{i, j} \pm \xi_{i}\left(\eta_{j}\right) \wedge \xi_{\bar{i}} \wedge \eta_{\bar{j}}
$$

where $\xi_{i}\left(\eta_{j}\right)=\xi_{i}^{a}\left(\partial_{a} \eta_{j}^{b}\right) \partial_{b}$. Note that, if one writes $\xi=\xi^{i_{0} \ldots i_{k}} \partial_{i_{0}} \wedge \cdots \wedge \partial_{i_{k}}$ and $\eta=\eta^{j_{0} \ldots j_{l}} \partial_{j_{0}} \wedge \cdots \wedge \partial_{j_{l}}$, the formula of the Nijenhuis-Richardson pre-Lie multiplication is written as (AMM02], p.19):

$$
\xi \bullet \eta=\sum_{r=1}^{k}(-1)^{r-1} \xi^{i_{0} \ldots i_{k}}\left(\partial_{i_{r}} \eta^{j_{0} \ldots j_{l}}\right) \partial_{i_{0}} \wedge \cdots \wedge \hat{\partial}_{i_{r}} \wedge \cdots \wedge \partial_{i_{k}} \wedge \partial_{j_{0}} \wedge \cdots \wedge \partial_{j_{l}},
$$

i.e., the Nijenhuis-Richardson multiplication can be written as an alternate sum of elementary multiplications $\bullet_{r}$, where

$$
\xi \bullet r \eta=\xi^{i_{0} \ldots i_{k}}\left(\partial_{i_{r}} \eta^{j_{0} \ldots j_{l}}\right) \partial_{i_{0}} \wedge \cdots \wedge \hat{\partial}_{i_{r}} \wedge \cdots \wedge \partial_{i_{k}} \wedge \partial_{j_{0}} \wedge \cdots \wedge \partial_{j_{l}} .
$$

For instance, if $\alpha \in T_{\text {poly }}^{1}$ and $\xi \in T_{\text {poly }}^{0}$, then

$$
\begin{aligned}
& \alpha \bullet \xi=\alpha^{i_{1} i_{2}}\left(\partial_{i_{1}} \xi^{i_{3}}\right) \partial_{i_{2}} \wedge \partial_{i_{3}}-\alpha^{i_{1} i_{2}}\left(\partial_{i_{2}} \xi^{i_{3}}\right) \partial_{i_{1}} \wedge \partial_{i_{3}} ; \\
& \xi \bullet \alpha=\xi^{i_{1}}\left(\partial_{i_{1}} \alpha^{i_{2} i_{3}}\right) \partial_{i_{2}} \wedge \partial_{i_{3}}
\end{aligned}
$$

Here is another example. If $\eta \in T_{\text {poly }}^{2}$ and $h \in T_{\text {poly }}^{-1}$, then

$$
\begin{aligned}
& \eta \bullet h=3 \eta^{i_{1} i_{2} i_{3}}\left(\partial_{i_{1}} h\right) \partial_{i_{2}} \wedge \partial_{i_{3}}, \\
& h \bullet \eta=0 .
\end{aligned}
$$

The trivial differential $d=0$ makes $T_{\text {poly }}^{\bullet}$ a differential graded Lie algebra. 
Remark 3.1. The above constructions can be globalized to a differential manifold $M$; in this case the differential graded Lie algebra of polyvector fields is denoted by

$$
T_{\text {poly }}^{\bullet}(M)=\bigoplus_{k=-1}^{\infty} T_{\text {poly }}^{k}(M)=\bigoplus_{k=-1}^{\infty} H^{0}\left(M, \wedge^{k+1} T M\right) .
$$

\subsection{The Lie algebra $D_{\text {poly }}^{\bullet}$}

The Hochschild complex of the algebra $\mathcal{C}^{\infty}\left(\mathbb{R}^{d}\right)$ of smooth functions on $\mathbb{R}^{d}$ is a differential graded Lie algebra whose underlying graded vector space is

$$
\begin{aligned}
\operatorname{Hoch}\left(\mathcal{C}^{\infty}\left(\mathbb{R}^{d}\right) ; \mathcal{C}^{\infty}\left(\mathbb{R}^{d}\right)\right) & =\bigoplus_{k=-1}^{\infty} \operatorname{Hoch}^{k}\left(\mathcal{C}^{\infty}\left(\mathbb{R}^{d}\right) ; \mathcal{C}^{\infty}\left(\mathbb{R}^{d}\right)\right) \\
& =\bigoplus_{k=-1}^{\infty} \operatorname{Hom}_{\mathbb{R}}\left(\mathcal{C}^{\infty}\left(\mathbb{R}^{d}\right)^{\otimes(k+1)}, \mathcal{C}^{\infty}\left(\mathbb{R}^{d}\right)\right)
\end{aligned}
$$

The Lie bracket is the Gerstenhaber bracket: if $\varphi \in \operatorname{Hoch}^{k}\left(\mathcal{C}^{\infty}\left(\mathbb{R}^{d}\right) ; \mathcal{C}^{\infty}\left(\mathbb{R}^{d}\right)\right)$ and $\psi \in \operatorname{Hoch}^{l}\left(\mathcal{C}^{\infty}\left(\mathbb{R}^{d}\right) ; \mathcal{C}^{\infty}\left(\mathbb{R}^{d}\right)\right)$, then the bracket $[\varphi, \psi]_{G}$ is the element of $\operatorname{Hoch}^{k+l}\left(\mathcal{C}^{\infty}\left(\mathbb{R}^{d}\right) ; \mathcal{C}^{\infty}\left(\mathbb{R}^{d}\right)\right)$ defined by

$$
\begin{aligned}
& \left\langle[\varphi, \psi]_{G} \mid f_{0} \otimes \cdots \otimes f_{k+l}\right\rangle= \\
& =\sum_{i=0}^{k}(-1)^{i l}\left\langle\varphi \mid f_{0} \otimes \cdots \otimes f_{i-1} \otimes\left\langle\psi \mid f_{i} \otimes \cdots f_{i+l}\right\rangle \otimes f_{i+l+1} \otimes \cdots \otimes f_{k+l}\right\rangle \\
& \quad-\sum_{i=0}^{l}(-1)^{k(l+i)}\left\langle\psi \mid f_{0} \otimes \cdots \otimes f_{i-1} \otimes\left\langle\varphi \mid f_{i} \otimes \cdots f_{i+k}\right\rangle \otimes f_{i+k+1} \otimes \cdots \otimes f_{k+l}\right\rangle
\end{aligned}
$$

As the Schouten-Nijenhuis bracket, the Gerstenhaber bracket is also induced by a pre-Lie multiplication, the Gerstenhaber composition

$$
\begin{aligned}
\circ: \operatorname{Hoch}^{k}\left(\mathcal{C}^{\infty}\left(\mathbb{R}^{d}\right) ; \mathcal{C}^{\infty}\left(\mathbb{R}^{d}\right)\right) \otimes \operatorname{Hoch}^{l}\left(\mathcal{C}^{\infty}\left(\mathbb{R}^{d}\right) ; \mathcal{C}^{\infty}\left(\mathbb{R}^{d}\right)\right) \rightarrow \\
\rightarrow \operatorname{Hoch}^{k+l}\left(\mathcal{C}^{\infty}\left(\mathbb{R}^{d}\right) ; \mathcal{C}^{\infty}\left(\mathbb{R}^{d}\right)\right)
\end{aligned}
$$

defined by

$$
\begin{aligned}
& \left\langle\varphi \circ \psi \mid f_{0} \otimes \cdots \otimes f_{k+l}\right\rangle= \\
& \quad=\sum_{i=0}^{k}(-1)^{i l}\left\langle\varphi \mid f_{0} \otimes \cdots \otimes f_{i-1} \otimes\left\langle\psi \mid f_{i} \otimes \cdots f_{i+l}\right\rangle \otimes f_{i+l+1} \otimes \cdots \otimes f_{k+l}\right\rangle
\end{aligned}
$$

It is immediate to see that

$$
[\varphi, \psi]_{G}=\varphi \circ \psi-(-1)^{k l} \psi \circ \varphi .
$$


Moreover, as for the Nijenhuis-Richardson multiplication • , the Gerstenhaber multiplication $\circ$ can also be written as a graded alternate sum of elementary compositions $\circ_{i}$, where

$$
\left\langle\varphi \circ_{i} \psi \mid f_{0} \otimes \cdots \otimes f_{k+l}\right\rangle=\left\langle\varphi \mid f_{0} \otimes \cdots \otimes f_{i-1} \otimes\left\langle\psi \mid f_{i} \otimes \cdots f_{i+l}\right\rangle \otimes f_{i+l+1} \otimes \cdots \otimes f_{k+l}\right\rangle
$$

If we denote by $\mu$ the multiplication of functions in $\mathcal{C}^{\infty}(M)$, then $\mu$ is an element in $\operatorname{Hoch}^{1}\left(\mathcal{C}^{\infty}\left(\mathbb{R}^{d}\right) ; \mathcal{C}^{\infty}\left(\mathbb{R}^{d}\right)\right)$ and the associativity of $\mu$ is equivalent to $[\mu, \mu]_{G}=0$. This means that $d_{\text {Hoch }}=[\mu, \cdot]_{G}$ is a degree one differential of $\operatorname{Hoch}^{\bullet}\left(\mathcal{C}^{\infty}\left(\mathbb{R}^{d}\right) ; \mathcal{C}^{\infty}\left(\mathbb{R}^{d}\right)\right)$. The differential $d_{\text {Hoch }}$ will be called the Hochschild differential. Explicitly, if $\varphi \in \operatorname{Hoch}^{k}\left(\mathcal{C}^{\infty}\left(\mathbb{R}^{d}\right) ; \mathcal{C}^{\infty}\left(\mathbb{R}^{d}\right)\right)$, then $d_{\text {Hoch }} \varphi$ is the element in $\operatorname{Hoch}^{k+1}\left(\mathcal{C}^{\infty}\left(\mathbb{R}^{d}\right) ; \mathcal{C}^{\infty}\left(\mathbb{R}^{d}\right)\right)$ defined by

$$
\begin{aligned}
& \left\langle d_{\text {Hoch }} \varphi \mid f_{0} \otimes \cdots \otimes f_{k+1}\right\rangle= \\
& =f_{0}\left\langle\varphi \mid f_{1} \otimes \cdots \otimes f_{k+1}\right\rangle-\sum_{i=0}^{k}\left\langle\varphi \mid f_{0} \otimes \cdots\left(f_{i} \cdot f_{i+1}\right) \otimes \cdots \otimes f_{k+1}\right\rangle \\
& \quad+(-1)^{k}\left\langle\varphi \mid f_{0} \otimes \cdots \otimes f_{k}\right\rangle f_{k+1} .
\end{aligned}
$$

For additional details see Kon97] §3.4.2 and [Cat04] 33.2 .2 .

One can define a cup-product

$$
\begin{aligned}
\cup: \operatorname{Hoch}^{k}\left(\mathcal{C}^{\infty}\left(\mathbb{R}^{d}\right) ; \mathcal{C}^{\infty}\left(\mathbb{R}^{d}\right)\right) \otimes \operatorname{Hoch}^{l}\left(\mathcal{C}^{\infty}\left(\mathbb{R}^{d}\right) ; \mathcal{C}^{\infty}\left(\mathbb{R}^{d}\right)\right) \rightarrow \\
\\
\rightarrow \operatorname{Hoch}^{k+l+1}\left(\mathcal{C}^{\infty}\left(\mathbb{R}^{d}\right) ; \mathcal{C}^{\infty}\left(\mathbb{R}^{d}\right)\right)
\end{aligned}
$$

as

$$
\left\langle D_{1} \cup D_{2} \mid f_{0} \cdots f_{k} \otimes f_{k+1} \cdots f_{k+l+1}\right\rangle=\left\langle D_{1} \mid f_{0} \otimes \cdots \otimes f_{k}\right\rangle \cdot\left\langle D_{2} \mid f_{k+1} \otimes \cdots \otimes f_{k+l+1}\right\rangle
$$

The space of polydifferential operators on $\mathbb{R}^{d}$ is the smallest subspace $D_{\text {poly }}^{\bullet}$ containing vector fields on $\mathbb{R}^{d}$ and the multiplications by smooth functions on $\mathbb{R}^{d}$, which is closed under the cup product and the o multiplication. More explicitly, elements of $D_{\text {poly }}^{k}$ are the linear operators on $\mathcal{C}^{\infty}\left(\mathbb{R}^{d}\right)^{\otimes(k+1)}$ with values in $\mathcal{C}^{\infty}\left(\mathbb{R}^{d}\right)$ which can be written as

$$
f_{0} \otimes \cdots \otimes f_{k} \mapsto C^{I_{0}, \ldots, I_{k}}\left(\partial_{I_{0}} f_{0}\right) \cdots\left(\partial_{I_{k}} f_{k}\right)
$$

for suitable multi-indices $I_{\bullet}$ and smooth functions $C^{I} \bullet$. It is immediate to see that $D_{\text {poly }}^{\bullet}$ is a differential graded Lie subalgebra of $\operatorname{Hoch}^{\bullet}\left(\mathcal{C}^{\infty}\left(\mathbb{R}^{d}\right) ; \mathcal{C}^{\infty}\left(\mathbb{R}^{d}\right)\right)$.

Remark 3.2. More in general, given a smooth manifold $M$ one can consider the differential graded Lie algebra $D_{\text {poly }}^{\bullet}(M)$ of polydifferential operators on $M$; it is a sub differential graded Lie algebra of $\operatorname{Hoch}^{\bullet}\left(\mathcal{C}^{\infty}(M), \mathcal{C}^{\infty}(M)\right)$.

\subsection{The Lie algebra $C E^{\bullet}\left(T_{\text {poly }}^{\bullet}, D_{\text {poly }}^{\bullet}\right)$}

In this section we define the Chevalley-Eilenberg differential graded Lie algebra $C E^{\bullet}\left(T_{\text {poly }}^{\bullet}, D_{\text {poly }}^{\bullet}\right)$. The construction we are going to describe is just a particular case of a more general construction of a differential graded Lie algebra 
$C E^{\bullet}\left(\mathfrak{g}_{1}, \mathfrak{g}_{2}\right)$ out of two differential graded Lie algebras $\mathfrak{g}_{1}$ and $\mathfrak{g}_{2}$, see [Kel] for hints and details.

As a bigraded vector space,

$$
\begin{aligned}
C E^{\bullet, \bullet}\left(T_{\text {poly }}^{\bullet}, D_{\text {poly }}^{\bullet}\right) & =\bigoplus_{p=-\infty}^{\infty} \bigoplus_{q=0}^{\infty} C E^{p, q}\left(T_{\text {poly }}^{\bullet}, D_{\text {poly }}^{\bullet}\right) \\
& =\bigoplus_{p=-\infty}^{\infty} \bigoplus_{q=0}^{\infty} \operatorname{Hom}\left(\wedge^{q} T_{\text {poly }}^{\bullet}, D_{\text {poly }}^{\bullet}[p]\right)
\end{aligned}
$$

where by $D_{\text {poly }}^{\bullet}[p]$ we mean the graded vector space $D_{\text {poly }}^{\bullet}$ with degrees shifted by $p$. The graded vector space $C E^{\bullet}\left(T_{\text {poly }}^{\bullet}, D_{\text {poly }}^{\bullet}\right)$ is the graded vector space associated with the bigraded space $C E^{\bullet \bullet \bullet}$, i.e.,

$$
\begin{aligned}
C E^{n}\left(T_{\text {poly }}^{\bullet}, D_{\text {poly }}^{\bullet}\right) & =\bigoplus_{p+q=n} C E^{p, q}\left(T_{\text {poly }}^{\bullet}, D_{\text {poly }}^{\bullet}\right) \\
& =\bigoplus_{q=0}^{\infty} \operatorname{Hom}\left(\wedge^{q} T_{\text {poly }}^{\bullet}, D_{\text {poly }}^{\bullet}[n-q]\right)
\end{aligned}
$$

The Lie bracket

$$
\begin{aligned}
{[,]_{C E}: C E^{p_{1}, q_{1}}\left(T_{\text {poly }}^{\bullet}, D_{\text {poly }}^{\bullet}\right) \otimes C E^{p_{2}, q_{2}} } & \left(T_{\text {poly }}^{\bullet}, D_{\text {poly }}^{\bullet}\right) \rightarrow \\
& \rightarrow C E^{p_{1}+p_{2}, q_{1}+q_{2}}\left(T_{\text {poly }}^{\bullet}, D_{\text {poly }}^{\bullet}\right)
\end{aligned}
$$

is defined by

$$
\begin{aligned}
& {[\mathcal{F}, \mathcal{H}]_{C E}\left(\gamma_{1} \wedge \cdots \wedge \gamma_{q_{1}+q_{2}}\right)=} \\
& \quad=\frac{1}{q_{1} ! q_{2} !} \sum_{\sigma \in \Sigma_{q_{1}+q_{2}}}\left[\mathcal{F}\left(\gamma_{\sigma(1)} \wedge \cdots \wedge \gamma_{\sigma\left(q_{1}\right)}\right), \mathcal{H}\left(\gamma_{\sigma\left(q_{1}+1\right)} \wedge \cdots \wedge \gamma_{\sigma\left(q_{1}+q_{2}\right)}\right)\right]_{G} .
\end{aligned}
$$

Being bigraded, the Lie bracket defined above induces a graded Lie bracket on $C E^{\bullet}\left(T_{\text {poly }}^{\bullet}, D_{\text {poly }}^{\bullet}\right)$. If we look at the multiplication $\mu$ on smooth functions on $\mathbb{R}^{d}$ as an element in $\left(D_{\text {poly }}^{\bullet}[1]\right)^{0}$, then the map $\mathcal{F}_{0}: 1 \mapsto \mu$ is an element in $C E^{1,0}\left(T_{\text {poly }}^{\bullet}, D_{\text {poly }}^{\bullet} \subseteq C E^{1}\left(T_{\text {poly }}^{\bullet}, D_{\text {poly }}^{\bullet}\right)\right.$. Since

$$
\left[\mathcal{F}_{0}, \mathcal{F}_{0}\right]_{C E}(1)=[\mu, \mu]_{G}=0,
$$

we have an horizontal differential

$$
d_{1}=\left[\mathcal{F}_{0}, \cdot\right]_{C E}: C E^{p, q}\left(T_{\text {poly }}^{\bullet}, D_{\text {poly }}^{\bullet}\right) \rightarrow C E^{p+1, q}\left(T_{\text {poly }}^{\bullet}, D_{\text {poly }}^{\bullet}\right)
$$

which makes $C E^{\bullet}\left(T_{\text {poly }}^{\bullet}, D_{\text {poly }}^{\bullet}\right)$ a differential graded Lie algebra. Explicitly, if $\mathcal{F} \in C E^{p, q}\left(T_{\text {poly }}^{\bullet}, D_{\text {poly }}^{\bullet}\right)$, then

$$
\left(d_{1} \mathcal{F}\right)\left(\gamma_{1} \wedge \cdots \wedge \gamma_{q}\right)=d_{\text {Hoch }}\left(\mathcal{F}\left(\gamma_{1} \wedge \cdots \wedge \gamma_{q}\right)\right) .
$$


We have not used the Lie algebra structure on $T_{\text {poly }}^{\bullet}$, yet. By looking at $D_{\text {poly }}^{\bullet}$ as

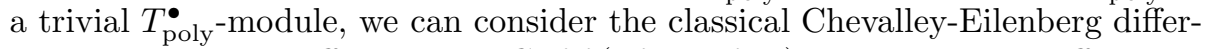
ential as a vertical differential on $C E^{\bullet \bullet \bullet}\left(T_{\text {poly }}^{\bullet}, D_{\text {poly }}^{\bullet}\right)$. Explicitly, the differential

$$
d_{2}: C E^{p, q}\left(T_{\text {poly }}^{\bullet}, D_{\text {poly }}^{\bullet}\right) \rightarrow C E^{p, q+1}\left(T_{\text {poly }}^{\bullet}, D_{\text {poly }}^{\bullet}\right)
$$

is defined by

$$
\left(d_{2} \mathcal{F}\right)\left(\gamma_{1} \wedge \cdots \wedge \gamma_{q+1}\right)=\sum_{i<j} \pm \mathcal{F}\left(\left[\gamma_{i}, \gamma_{j}\right]_{S N} \wedge \gamma_{1} \wedge \cdots \wedge \widehat{\gamma_{i}} \wedge \cdots \wedge \widehat{\gamma_{j}} \wedge \cdots \wedge \gamma_{q+1}\right)
$$

By looking at an expression of the form

$$
\left\langle\mathcal{F}\left(\gamma_{1} \wedge \cdots \wedge \gamma_{n}\right) \mid f_{0} \otimes \cdots \otimes f_{m}\right\rangle
$$

one sees that $d_{1}$ acts on the right side of the pairing whereas $d_{2}$ acts on the left side. As a consequence, the two differentials commute, and define a degree one total differential $d_{C E}=d_{1} \pm d_{2}$ making $C E^{\bullet}\left(T_{\text {poly }}^{\bullet}, D_{\text {poly }}^{\bullet}\right)$ a differential graded Lie algebra.

\section{The Maurer-Cartan equation and $L_{\infty}$-morphisms}

Recall that Kontsevich's approach consists in pushing forward a solution of the Maurer-Cartan equation in $T_{\text {poly }}^{\bullet}$ (the Poisson structure $\alpha$ ) via an $L_{\infty}$-morphism $\mathcal{F}: T_{\text {poly }}^{\bullet} \rightarrow D_{\text {poly }}^{\bullet}$, to get a solution of the Maurer-Cartan equation in $D_{\text {poly }}^{\bullet}$ (the star-product). In this section we explain how $L_{\infty}$-morphisms correspond to solutions of the Maurer-Cartan equation in the Chevalley-Eilenberg differential graded Lie algebra $C E^{\bullet}\left(T_{\text {poly }}^{\bullet}, D_{\text {poly }}^{\bullet}\right)$ and how each of these solutions leads to an associative star-product quantizing a given Poisson structure.

\subsection{The Maurer-Cartan equation on the Chevalley-Eilen- berg complex}

Given a differential graded Lie algebra $\mathfrak{g}=\bigoplus_{i=0}^{\infty} \mathfrak{g}^{i}$, the Maurer-Cartan equation for $\mathfrak{g}$ is the following equation for an element $\gamma$ in $\mathfrak{g}^{1}$ :

$$
d \gamma+\frac{1}{2}[\gamma, \gamma]=0
$$

The set of the solutions of the Maurer-Cartan equation for $\mathfrak{g}$ will be denoted by $M C(\mathfrak{g})$. We are interested in the set $M C\left(C E^{\bullet}\left(T_{\text {poly }}^{\bullet}, D_{\text {poly }}^{\bullet}\right)\right)$. Since

$$
\begin{aligned}
C E^{1}\left(T_{\text {poly }}^{\bullet}, D_{\text {poly }}^{\bullet}\right) & =\bigoplus_{p+q=1} C E^{p, q}\left(T_{\text {poly }}^{\bullet}, D_{\text {poly }}^{\bullet}\right) \\
& =\bigoplus_{q=0}^{\infty} \operatorname{Hom}\left(\wedge^{q} T_{\text {poly }}^{\bullet}, D_{\text {poly }}^{\bullet}[1-q]\right),
\end{aligned}
$$


a solution of the Maurer-Cartan equation for the Chevalley-Eilenberg complex of $T_{\text {poly }}^{\bullet}$ and $D_{\text {poly }}^{\bullet}$ is

$$
\mathcal{F}=\mathcal{F}_{0}+\mathcal{F}_{1}+\mathcal{F}_{2}+\cdots
$$

where

$$
\mathcal{F}_{q}: \wedge^{q} T_{\text {poly }}^{\bullet} \rightarrow D_{\text {poly }}^{\bullet}[1-q]
$$

and the $\mathcal{F}_{q}$ 's satisfy the equations

$$
d_{1} \mathcal{F}_{q} \pm d_{2} \mathcal{F}_{q-1}+\frac{1}{2} \sum_{q_{1}+q_{2}=q}\left[\mathcal{F}_{q_{1}}, \mathcal{F}_{q_{2}}\right]_{C E}=0, \quad q \geq 0,
$$

corresponding to the $\operatorname{Hom}\left(\wedge^{q} T_{\text {poly }}^{\bullet}, D_{\text {poly }}^{\bullet}[2-q]\right)$-components of the single MaurerCartan equation

$$
d_{C E} \mathcal{F}+\frac{1}{2}[\mathcal{F}, \mathcal{F}]_{C E}=0 .
$$

In particular $\mathcal{F}_{0}$ has to satisfy the equation $d_{\text {Hoch }}\left(\mathcal{F}_{0}(1)\right)+\frac{1}{2}\left[\mathcal{F}_{0}(1), \mathcal{F}_{0}(1)\right]_{G}=0$, that is $\mu+\mathcal{F}_{0}(1)$ has to be an associative product on the space of smooth functions on $\mathbb{R}^{d}$. This equation is trivially satisfied if we choose $\mathcal{F}_{0}=0$ : as usual, one should think of solutions of the Maurer-Cartan equations as perturbations; when added to the base point (the "initial value") they yield the desired deformations.

If we consider the space $M C_{0}\left(C E^{\bullet}\left(T_{\text {poly }}^{\bullet}, D_{\text {poly }}^{\bullet}\right)\right)$ of solutions of the MaurerCartan equation on the Chevalley-Eilenberg complex of $T_{\text {poly }}^{\bullet}$ and $D_{\text {poly }}^{\bullet}$ such that the "initial value" $\mathcal{F}_{0}$ is zero, then the equations for an element $\mathcal{F}=$ $\mathcal{F}_{0}+\mathcal{F}_{1}+\mathcal{F}_{2}+\cdots$ of $M C_{0}\left(C E^{\bullet}\left(T_{\text {poly }}^{\bullet}, D_{\text {poly }}^{\bullet}\right)\right)$ are

$$
\left\{\begin{array}{l}
\mathcal{F}_{0}=0 \\
d_{1} \mathcal{F}_{q} \pm d_{2} \mathcal{F}_{q-1}+\frac{1}{2} \sum_{\substack{q_{1}+q_{2}=q \\
q_{1}, q_{2} \geq 1}}\left[\mathcal{F}_{q_{1}}, \mathcal{F}_{q_{2}}\right]_{C E}=0, \quad q \geq 1
\end{array}\right.
$$

Written out explicitly for $q$ polyvector fields $\gamma_{1}, \ldots \gamma_{q}$, these equations are

$$
\begin{aligned}
& d_{\text {Hoch }}\left(\mathcal{F}_{q}\left(\gamma_{1} \wedge \cdots \wedge \gamma_{q}\right)\right)+\sum_{i<j} \pm \mathcal{F}_{q-1}\left(\left[\gamma_{i}, \gamma_{j}\right]_{S N} \wedge \gamma_{1} \wedge \cdots \wedge \widehat{\gamma_{i}} \wedge \cdots \wedge \widehat{\gamma}_{j} \wedge \cdots \wedge \gamma_{q}\right)+ \\
+ & \frac{1}{2} \sum_{\substack{q_{1}+q_{2}=q \\
q_{1}, q_{2} \geq 1}} \frac{1}{q_{1} ! q_{2} !} \sum_{\sigma \in \Sigma_{q}} \pm\left[\mathcal{F}_{q_{1}}\left(\gamma_{\sigma(1)} \wedge \cdots \wedge \gamma_{\sigma\left(q_{1}\right)}\right), \mathcal{F}_{q_{2}}\left(\gamma_{\sigma\left(q_{1}+1\right)} \wedge \cdots \wedge \gamma_{\sigma(q)}\right)\right]_{G}=0
\end{aligned}
$$

where $\Sigma_{q}$ denotes the symmetric group on $q$ elements. But this precisely means that the maps $\mathcal{F}_{q}: \wedge^{q} T_{\text {poly }}^{\bullet} \rightarrow D_{\text {poly }}^{\bullet}[1-q], q \geq 1$ are the Taylor coefficients of an $L_{\infty}$-morphism between the differential graded Lie algebras $T_{\text {poly }}^{\bullet}$ and $D_{\text {poly }}^{\bullet}$. We have therefore proved the following equivalence.

Proposition 4.1. The set $\operatorname{Hom}_{L_{\infty}}\left(T_{\text {poly }}^{\bullet}, D_{\text {poly }}^{\bullet}\right)$ is naturally identified with the set $M C_{0}\left(C E^{\bullet}\left(T_{\text {poly }}^{\bullet}, D_{\text {poly }}^{\bullet}\right)\right)$ of solutions of the Maurer-Cartan equation in the Chevalley-Eilenberg differential graded Lie algebra $C E^{\bullet}\left(T_{\text {poly }}^{\bullet}, D_{\text {poly }}^{\bullet}\right)$ of $T_{\text {poly }}^{\bullet}$ and $D_{\text {poly }}^{\bullet}$, such that $\mathcal{F}_{0}=0$. 


\subsection{The quantization of Poisson structures}

Let now $\mathcal{F}$ be an element of $M C_{0}\left(C E^{\bullet}\left(T_{\text {poly }}^{\bullet}, D_{\text {poly }}^{\bullet}\right)\right)$, and let $\alpha$ be a Poisson structure on $\mathbb{R}^{d}$. Then $\alpha$ is an element of $T_{\text {poly }}^{1}$ and $[\alpha, \alpha]_{S N}=0$. Since $\alpha$ lies in $T_{\text {poly }}^{1}, \mathcal{F}_{q}\left(\alpha^{\wedge q}\right)$ is an element of $D_{\text {poly }}^{1}$ for any $q \geq 0$. It follows that the formal power series

$$
\pi_{\alpha}(\hbar):=\mathcal{F}(\exp \{\hbar \alpha\})
$$

is an element of $D_{\text {poly }}^{1}[[\hbar]]$. Choosing $\gamma_{i}=\alpha$ for any $i$ from 1 to $q$ in the equations for the $\mathcal{F}_{q}$ 's written above, we find

$$
d_{\text {Hoch }}\left(\mathcal{F}_{q}\left(\alpha^{\wedge q} / q !\right)\right)+\frac{1}{2} \sum_{\substack{q_{1}+q_{2}=q \\ q_{1}, q_{2} \geq 1}} \pm\left[\mathcal{F}_{q_{1}}\left(\alpha^{\wedge q_{1}} / q_{1} !\right), \mathcal{F}_{q_{2}}\left(\alpha^{\wedge q_{2}} / q_{2} !\right)\right]_{G}=0 .
$$

Therefore

$$
d_{\mathrm{Hoch}} \pi_{\alpha}+\frac{1}{2}\left[\pi_{\alpha}, \pi_{\alpha}\right]_{G}=0
$$

In other words,

$$
\mu_{\alpha}(\hbar):=\mu+\pi_{\alpha}
$$

is an associative product (depending on the formal parameter $\hbar$ ) on the space of the smooth functions on $\mathbb{R}^{d}$. Note that $\lim _{\hbar \rightarrow 0} \mu_{\alpha}(\hbar)=\mu$, i.e., $\mu_{\alpha}(\hbar)$ is an associative deformation of $\mu$. For two smooth functions $f$ and $g$ on $\mathbb{R}^{d}$, we set

$$
f \star_{\alpha} g:=\left\langle\mu_{\alpha}(\hbar) \mid f \otimes g\right\rangle=f g+\hbar\left\langle\mathcal{F}_{1}(\alpha) \mid f \otimes g\right\rangle+O\left(\hbar^{2}\right) .
$$

As an immediate consequence, we obtain

Proposition 4.2. Any solution $\mathcal{F}$ of the Maurer-Cartan equation on the Chevalley-Eilenberg DGLA $C E^{\bullet}\left(T_{\text {poly }}^{\bullet}, D_{\text {poly }}^{\bullet}\right.$ ) with $\mathcal{F}_{0}=0$ and $\mathcal{F}_{1}(\gamma)=\gamma$ (with the usual identification of polyvector fields with polydifferential operators) leads to $a$ star product $\star_{\alpha}$ such that

$$
f \star_{\alpha} g=f g+\hbar\{f, g\}_{\alpha}+O\left(\hbar^{2}\right),
$$

i.e., to a deformation quantization of the Poisson structure $\alpha$.

\subsection{The vertical Maurer-Cartan equation}

It is convenient to consider the translation of $\mathcal{F}$ by $\mu$, since the defining equations for $\mathcal{F}^{\mu}=\mu+\mathcal{F}$ are simpler than the original equations for $\mathcal{F}$. In particular, $\mathcal{F}^{\mu}$ can be seen as a solution of the "vertical" Maurer-Cartan equation in the Chevalley-Eilenberg complex, i.e., $\mathcal{F}^{\mu}$ satisfies

$$
d_{2} \mathcal{F}^{\mu}+\frac{1}{2}\left[\mathcal{F}^{\mu}, \mathcal{F}^{\mu}\right]_{C E}=0
$$

Indeed, the components of $\mathcal{F}^{\mu}$ are given by

$$
\left\{\begin{array}{l}
\mathcal{F}_{0}^{\mu}(1)=\mu+\mathcal{F}_{0}(1) \\
\mathcal{F}_{q}^{\mu}=\mathcal{F}_{q} ; \text { for } q \geq 1
\end{array}\right.
$$


and we can rewrite the equations for $\mathcal{F}$ as

$$
\left\{\begin{array}{l}
\mathcal{F}_{0}^{\mu}(1)=\mu \\
d_{1} \mathcal{F}_{q}^{\mu} \pm d_{2} \mathcal{F}_{q-1}^{\mu}+\frac{1}{2} \sum_{\substack{q_{1}+q_{2}=q \\
q_{1}, q_{2} \geq 1}}\left[\mathcal{F}_{q_{1}}^{\mu}, \mathcal{F}_{q_{2}}^{\mu}\right]_{C E}=0, \quad q \geq 1
\end{array}\right.
$$

where we have used the fact that $d_{2} \mathcal{F}_{0}^{\mu}$ is trivially zero since the "vertical" differential $d_{2}$ is zero on $C E^{p, 0}\left(T_{\text {poly }}^{\bullet}, D_{\text {poly }}^{\bullet}\right)$. Since the "horizontal" differential $d_{1}$ on the Chevalley-Eilenberg complex coincides with the Lie-adjoint of $\mathcal{F}_{0}^{\mu}$, we can rewrite the above equations as

$$
\left\{\begin{array}{l}
\mathcal{F}_{0}^{\mu}(1)=\mu \\
d_{2} \mathcal{F}_{q-1}^{\mu}+\frac{1}{2} \sum_{\substack{q_{1}+q_{2}=q \\
q_{1}, q_{2} \geq 0}}\left[\mathcal{F}_{q_{1}}^{\mu}, \mathcal{F}_{q_{2}}^{\mu}\right]_{C E}=0, \quad q \geq 1
\end{array}\right.
$$

which is precisely the vertical Maurer-Cartan equation on $C E^{\bullet}\left(T_{\text {poly }}^{\bullet}, D_{\text {poly }}^{\bullet}\right)$.

Remark 4.1. The space $M C_{\mu}\left(C E^{\bullet}\left(T_{\text {poly }}^{\bullet}, D_{\text {poly }}^{\bullet}\right), d_{2}\right)$ of solutions of the vertical Maurer-Cartan equation with $\mathcal{F}_{0}^{\mu}(1)=\mu$ should be thought as an affine version of $M C_{0}\left(C E^{\bullet}\left(T_{\text {poly }}^{\bullet}, D_{\text {poly }}^{\bullet}\right), d_{C E}\right)$. In particular, the translation by $\mu$ establishes a one to one correspondence between $M C_{0}\left(C E^{\bullet}\left(T_{\text {poly }}^{\bullet}, D_{\text {poly }}^{\bullet}\right), d_{C E}\right)$ and $M C_{\mu}\left(C E^{\bullet}\left(T_{\text {poly }}^{\bullet}, D_{\text {poly }}^{\bullet}\right), d_{2}\right)$. As a consequence, we get a star-product quantization of Poisson structures out of any element of $M C_{\mu}\left(C E^{\bullet}\left(T_{\text {poly }}^{\bullet}, D_{\text {poly }}^{\bullet}\right), d_{2}\right)$ such that $\mathcal{F}_{1}^{\mu}: T_{\text {poly }}^{m} \rightarrow D_{\text {poly }}^{m}$ is the canonical identification of polyvector fields with polydifferential operators.

\section{The differential graded Lie algebra of graphs}

Kontsevich's proof from Kon97 clearly hints to a coproduct structure controlling the coefficients of the formality morphism Ion03. The corresponding result was stated in Ion04b in a framework dual to the DGLA picture briefly mentioned in Ion04a. Here we focus on the DGLA structure with its two pre-Lie operations inspired by Connes-Kreimer's insertion operation and Gerstenhaber composition.

Recall from section 2.1 that $\mathcal{G}^{p, q}$ is the set of isomorphism classes of all admissible graphs $\Gamma$ with $q$ internal vertices and such that $v(\Gamma)-e(\Gamma)-1=p$, where $v(\Gamma)$ and $e(\Gamma)$ are the number of vertices and edges of $\Gamma$, respectively. Finally,

$$
\mathscr{G}^{\bullet}, \bullet=\bigoplus_{p, q=0}^{\infty} \mathscr{G}^{p, q}
$$

will denote the bigraded vector space generated by $\mathcal{G}^{\bullet} \bullet=\cup_{p, q=0}^{\infty} \mathcal{G}^{p, q}$. 
If $\Phi$ is a subgraph of $\Gamma$, we can collapse $\Phi$ to a point, obtaining a new graph $\Gamma / \Phi$; the point corresponding to the collapsed subgraph will be a vertex $v_{\Phi}$ in the quotient graph, which can be either an internal or a boundary vertex. To describe more precisely the collapsing of subgraphs, recall that admissible graphs have to be thought as embedded into a disk. To collapse a subgraph $\Phi$ to a point, one first chooses a point $v_{\Phi}$ in the disk; then one considers a simply connected region $R_{\Phi}$ in the disk having a connected (possibly empty) intersection with the boundary of the disk and which contains $v_{\Phi}$ and all the vertices of $\Phi$, but contains no vertex of $\Gamma$ which is not in $\Phi$. Note that this requirement implies that not every subgraph of $\Gamma$ can be contracted to a point: for instance, if $\Phi$ contains two boundary vertices $v_{1}$ and $v_{2}$ then, to be a contractible subgraph, $\Phi$ has to contain also all the boundary vertices of $\Gamma$ which lie between $v_{1}$ and $v_{2}$. Then one collapses the whole region $R_{\Phi}$ to the point $v_{\Phi}$ is such a way that, during the collapsing, the points of the region which were on the boundary of the disk remain constrained on the boundary of the disk. Note that this implies that $v_{\Phi}$ is forced to be a boundary vertex if $\Phi$ contains at least a boundary vertex.

The set of admissible graphs is not closed under the operation of quotienting by contractible subgraphs, i.e., given an admissible graph $\Gamma$ and a contractible subgraph $\Phi$ of $\Gamma$, the quotient graph $\Gamma / \Phi$ is not necessarily an element of $\mathcal{G}^{\bullet} \bullet \bullet$. For instance, in the graph $\Gamma / \Phi$ there could be a loop or an edge stemming from a boundary vertex.

Definition 5.1. We say that the subgraph $\Phi$ is a normal subgraph of $\Gamma$ if $\Gamma / \Phi$ is an element of $\mathcal{G}^{\bullet} \bullet$; in such a case we write $\Phi \preccurlyeq \Gamma$.

Remark 5.1. Note that a normal subgraph $\Phi \leqslant \Gamma$ has to be a full subgraph, i.e. every edge of $\Gamma$ connecting two vertices of $\Phi$ is an edge of $\Phi$. Indeed, if $\Phi$ were not full, then there would be two vertices $v_{1}$ and $v_{2}$ in $\Phi$ and an edge $e$ joining $v_{1}$ and $v_{2}$ in $\Gamma$ which is not an edge in $\Phi$. Then, in the quotient graph $\Gamma / \Phi$ the edge $e$ would be a loop starting and ending on the vertex $v_{\Phi}$, and so $\Gamma / \Phi$ would not be admissible.

Definition 5.2. If $\Phi$ is a normal subgraph of $\Gamma$ and $\Gamma / \Phi=\Psi$, we say that $\Gamma$ is an extension of $\Psi$ by $\Phi$ and write

$$
\Phi \hookrightarrow \Gamma \rightarrow \Psi
$$

This simply means that $\Gamma$ is obtained by inserting the graph $\Phi$ into a vertex of $\Psi$. Depending on the type of vertex $\Phi$ is inserted into, the extension is called internal or boundary extension.

Note that given two graphs $\Phi$ and $\Psi$ we cannot talk of "the extension" of $\Psi$ by $\Phi$ since there are many possible extensions, even if one fixes the vertex of $\Psi$ where to insert $\Phi$. On the other hand, the sum of all possible extensions of $\Psi$ by $\Phi$ is a well-defined element of the vector space $\mathscr{G} \bullet \bullet \bullet$, so we get a bilinear operation on $\mathscr{G} \bullet \bullet$. Depending whether we consider all extensions or only 
internal/boundary extensions, we obtain the Connes-Kreimer product

$$
\Psi * \Phi=\sum_{\Phi \hookrightarrow \Gamma \rightarrow \Psi} \pm \Gamma
$$

or the Hochschild-Kontsevich products

$$
\begin{aligned}
& \Psi \bullet \Phi=\sum_{\substack{\Phi \hookrightarrow \Gamma \rightarrow \Psi \\
\text { internal }}} \pm \Gamma \\
& \Psi \circ \Phi=\sum_{\substack{\Phi \hookrightarrow \Gamma \rightarrow \Psi \\
\text { boundary }}} \pm \Gamma
\end{aligned}
$$

The signs in the above formulas depend upon the combinatorial data of the graphs involved. Explicit formulas can be found, for instance, in AMM02. Note that the product $\circ$ above is bigraded:

$$
\circ: \mathscr{G}^{p_{1}, q_{1}} \otimes \mathscr{G}^{p_{2}, q_{2}} \rightarrow \mathscr{G}^{p_{1}+p_{2}, q_{1}+q_{2}},
$$

whereas the $\bullet$ product has degree $(0,-1)$ :

$$
\bullet: \mathscr{G}^{p_{1}, q_{1}} \otimes \mathscr{G}^{p_{2}, q_{2}} \rightarrow \mathscr{G}^{p_{1}+p_{2}, q_{1}+q_{2}-1} .
$$

The Connes-Kreimer product $*$, being a combination of $\circ$ and $\bullet$, is non homogeneous. In the rest of this paper we will be interested only in the HochschildKontsevich products and the Lie algebra structures they induce; the reader interested in Connes-Kreimer product is referred to [CK98.

\subsection{The $\circ$ composition of graphs}

It is a simple check to see that the $\circ$ operation makes $\mathscr{G} \bullet \bullet \bullet$ a pre-Lie algebra, i.e. the (graded) commutator defines a bigraded Lie algebra structure on $\mathscr{G} \bullet \bullet$.

As for the Gerstenhaber composition, also the graph composition $\circ$ can be written as an alternate sum of elementary compositions

$$
\begin{aligned}
\circ_{i}: \mathscr{G}^{p_{1}, q_{1}} \otimes \mathscr{G}^{p_{1}, q_{1}} & \rightarrow \mathscr{G}^{p_{1}+p_{2}, q_{1}+q_{2}} \\
\Psi \otimes \Phi & \mapsto \Psi \circ_{i} \Phi,
\end{aligned}
$$

which insert the graph $\Phi$ in the $i^{\text {th }}$ boundary vertex of the graph $\Psi$ ("a la Kathotia" Kat98, p.15: a leg of $\Psi$ may land on any vertex of $\Phi$; as we will see in the next section this corresponds to Leibniz rule for derivations). As far as concerns the labeling of the edges and vertices of $\Psi \circ \Phi$, the labeling of internal vertices of $\Psi \circ_{i} \Phi$ is defined as the concatenation of the corresponding labelings, the labeling of boundary vertices of $\Psi \circ_{i} \Phi$ is defined "inserting" the boundary labels of $\Phi$ shifted by $i-1$ in place of the $i^{\text {th }}$ boundary vertex of $\Psi$ and consequently shifting the labels on the other boundary vertices of $\Psi$; the total order on edges from each internal vertex is unchanged. Here is an example. 


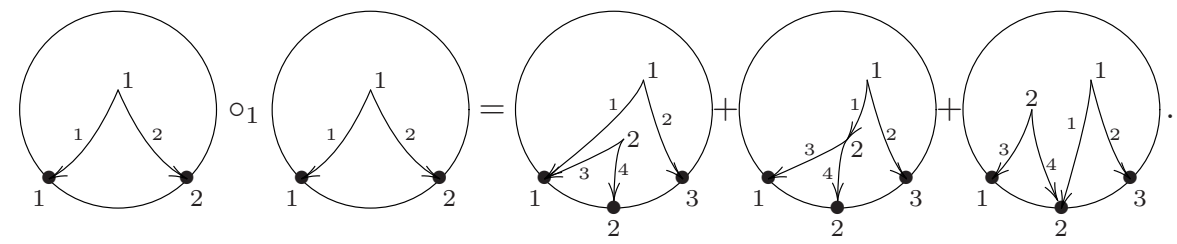

We will denote by [, ] the corresponding Lie bracket

$$
[\Psi, \Phi]=\Psi \circ \Phi \pm \Phi \circ \Psi
$$

and will call it the Hochschild-Kontsevich Lie bracket. Note that the graph $b_{0,2}$ with two boundary vertices and no internal vertices satisfies $\left[b_{0,2}, b_{0,2}\right]=0$. Therefore it induces a differential $d_{1}=\left[b_{0,2}, \cdot\right]$ on $\mathscr{G}^{\bullet \bullet \bullet}$. Since $b_{0,2} \in \mathscr{G}^{1,0}$ and the Hochschild-Kontsevich Lie bracket is bigraded, $d_{1}$ is a degree $(1,0)$ differential:

$$
d_{1}: \mathscr{G}^{p, q} \rightarrow \mathscr{G}^{p+1, q} .
$$

\subsection{The differential $d_{2}$ on graphs}

The "vertical" differential $d_{2}$ on admissible graphs is the usual graph homology differential introduced by Kontsevich in Kon96, p.151 (see also Vor, p.3): $d_{2} \Psi$ is the alternate sum over all graphs $\Gamma$ which can be obtained by expanding the internal vertices of $\Psi$ by the insertion of an additional edge. In formulas,

$$
d_{2} \Psi=\sum_{\substack{e \leftrightarrow \Gamma \rightarrow \Psi \\ \text { internal }}} \pm \Gamma
$$

This is conveniently rewritten in terms of the $\bullet$ multiplication we described above:

$$
d_{2} \Psi=\Psi \bullet e
$$

The usual argument shows that $d_{2}$ is indeed a differential: $(\Psi \bullet e) \bullet e$ is basically the sum over all ways of inserting two edges in the internal vertices of $\Psi$, and each term in this sum occurs twice with opposite signs. Note that, since the $\bullet$ multiplication has degree $(0,-1)$ and $e \in \mathscr{G}^{0,2}$, the differential $d_{2}$ is a degree $(0,1)$ differential.

As the $\circ$ multiplication, also the $\bullet$ multiplication can be written as an alternate sum of elementary $\bullet_{i}$ operations, which insert the second graph in the $i^{\text {th }}$ internal vertex of the first graph. This remark leads to the description of the differential $d_{2}$ acting on admissible graphs as $d_{2}=\sum_{i} \pm d_{2, i}$, where $d_{2, i}: \mathscr{G}^{p, q} \rightarrow \mathscr{G}^{p, q+1}$ is the operator which inserts an edge (both ways) splitting the $i^{\text {th }}$ vertex of a graph. More precisely, $d_{2, i} \Psi=\sum \pm \Gamma$, where the sum ranges over the set of graphs $\Gamma$ which are obtained by inserting an edge (both ways) in the $i^{\text {th }}$ vertex of $\Psi$.

Here is an example. 


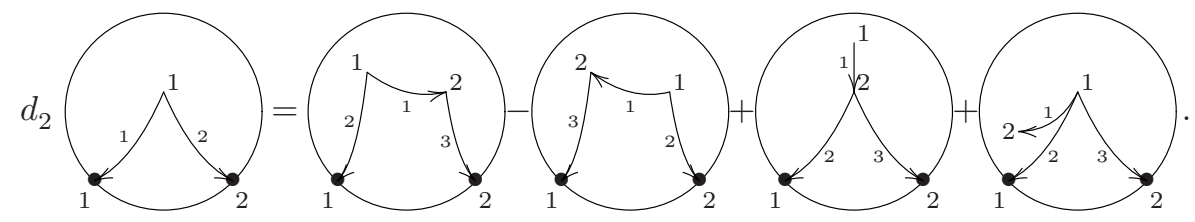

\subsection{The total differential on graphs}

We have shown that there are two differentials on the bi-graded vector space $\mathscr{G} \bullet \bullet$. Namely, we have the degree $(1,0)$ differential $d_{1}$ an the degree $(0,1)$ differential $d_{2}$. We will now show that the two differentials commute, so that they induce a degree one total differential $d=d_{1} \pm d_{2}$ on the total graph complex $\mathscr{G} \bullet$, where

$$
\mathscr{G}^{n}=\bigoplus_{p+q=n} \mathscr{G}^{p, q}
$$

Moreover, the total differential $d$ will turn out to be compatible with the graded Lie bracket [, ] on $\mathscr{G} \bullet$, so that we obtain a differential graded Lie algebra of admissible graphs. We summarize these results in the following

Proposition 5.1. The Lie bracket [, ] and the differential d endow the graded vector space $\mathscr{G} \bullet$ with a differential graded Lie algebra structure, which we will call the Hochschild-Kontsevich differential graded Lie algebra of graphs.

Proof. Since the differential $d_{1}$ is defined by means of the Lie bracket [, ] on $\mathscr{G} \bullet$, and $d_{2} b_{0,2}=0$, the compatibility of the differential $d_{2}$ with the Lie bracket will imply at once both the compatibility of the two differentials and the compatibility of the total differential with the Lie bracket. Since the internal vertices of $\Psi \circ \Phi$ are exactly the disjoint union of the internal vertices of $\Phi$ and of the internal vertices of $\Psi$, we have

$$
(\Psi \circ \Phi) \bullet e=(\Psi \bullet e) \circ \Phi+\Psi \circ(\Phi \bullet e),
$$

which immediately implies the compatibility of $d_{2}$ with the Lie bracket.

Remark 5.2. The DGLA structure on graphs we have described is actually part of a richer algebraic structure. Indeed, juxtaposition of graphs defines a degree $(-1,0)$ operation $\cup: \mathscr{G}^{p_{1}, q_{1}} \otimes \mathscr{G}^{p_{2}, q_{2}} \rightarrow \mathscr{G}^{p_{1}+p_{2}-1, q_{1}+q_{2}}$ which, together with the Lie bracket [, ], satisfies the axioms of Gerstenhaber algebra, up to homotopy. On the $T_{\text {poly }}^{\bullet}$-side, the $\cup$ multiplication corresponds to the usual wedge product of polyvector fields; on the $D_{\text {polv }}^{\bullet}$-side it corresponds to the cup-product on the Hochschild complex. See GaH03 and GiH03 for details. 


\section{The map $\mathcal{U}$ as a morphism of differential gra- ded Lie algebras}

Recall from Section 2 that the graphical calculus for derivations can be seen as a map

$$
\mathcal{U}: \mathcal{G}^{p, q} \rightarrow \operatorname{Hom}\left(\left(T_{\text {poly }}^{\bullet}\right)^{\otimes q}, D_{\text {poly }}^{\bullet}[p]\right),
$$

Therefore, if we identify the antisymmetric product $\Lambda^{q} T_{\text {poly }}^{\bullet}$ with a subspace of the tensor product $\otimes^{q} T_{\text {poly }}^{\bullet}$ via the usual anti-symmetrization map, we can look at $\mathcal{U}$ as a map of bigraded vector spaces

$$
\mathcal{U}: \mathscr{G}^{p, q} \rightarrow C E^{p, q}\left(T_{\text {poly }}^{\bullet}, D_{\text {poly }}^{\bullet}\right) .
$$

We will show in this section that $\mathcal{U}$ is actually a map of differential graded Lie algebras.

Theorem 6.1. The graphical calculus for derivations $\mathcal{U}$ is a morphism of differential graded Lie algebras.

The proof will be divided into two parts.

\section{1 $\mathcal{U}$ as a morphism of Lie algebras}

Lemma 6.1. $\left[\mathcal{U}_{\Gamma_{1}}, \mathcal{U}_{\Gamma_{2}}\right]_{C E}=\mathcal{U}_{\left[\Gamma_{1}, \Gamma_{2}\right]}$

Proof. Let $\Gamma_{1} \in \mathscr{G}^{p_{1}, q_{1}}$ and $\Gamma_{2} \in \mathscr{G}^{p_{2}, q_{2}}$. The lemma will immediately follow from the finer statement

$$
\mathcal{U}_{\Gamma_{1}}\left(\xi_{1} \otimes \cdots \otimes \xi_{q_{1}}\right) \circ_{i} \mathcal{U}_{\Gamma_{2}}\left(\xi_{q_{1}+1} \otimes \cdots \otimes \xi_{q_{1}+q_{2}}\right)=\mathcal{U}_{\Gamma_{1} \circ_{i} \Gamma_{2}}\left(\xi_{1} \otimes \cdots \otimes \xi_{q_{1}+q_{2}}\right),
$$

where the $\circ_{i}$ operation on the left is the $i^{\text {th }}$ insertion in the Gerstenhaber preLie operation $\circ$, and the $\circ_{i}$ operation the right is the operation that inserts the graph $\Gamma_{2}$ in the $i^{\text {th }}$ boundary vertex of the graph $\Gamma_{1}$. By definition of the $\circ_{i}$ Gerstenhaber composition, what we have to show is that

$$
\begin{aligned}
\left\langle\mathcal{U}_{\Gamma_{1} \circ_{i} \Gamma_{2}}\left(\xi_{1} \otimes \cdots \otimes \xi_{q_{1}+q_{2}}\right) \mid f_{0} \otimes \cdots \otimes f_{m_{1}+m_{2}-2}\right\rangle & \\
= & \left\langle\mathcal{U}_{\Gamma_{1}}\left(\xi_{1} \otimes \cdots \otimes \xi_{q_{1}}\right)\right| f_{0} \otimes \cdots \otimes f_{i-1} \otimes \\
& \otimes\left\langle\mathcal{U}_{\Gamma_{2}}\left(\xi_{q_{1}+1} \otimes \cdots \otimes \xi_{q_{1}+q_{2}}\right) \mid f_{i} \otimes \cdots \otimes f_{i+m_{2}-1}\right\rangle \otimes \\
& \left.\otimes f_{i+m_{1}} \otimes \cdots \otimes f_{m_{1}+m_{2}-2}\right\rangle,
\end{aligned}
$$

where $m_{1}$ and $m_{2}$ are the number of boundary vertices of $\Gamma_{1}$ and $\Gamma_{2}$, respectively. This directly follows from the definition of the $\circ_{i}$ graph boundary insertion and by the Leibniz rule for derivations.

Example 6.1. We will check that $b_{1,2} \circ_{1} b_{1,2}$ is mapped by $\mathcal{U}$ to $\mathcal{U}_{b_{1,2}} \circ_{1} \mathcal{U}_{b_{1,2}}$, where $b_{1,2}$ is the 2 -corolla

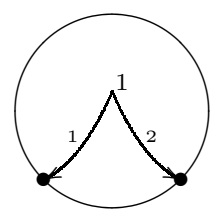


Clearly, $\mathcal{U}_{b_{1,2}}\left(\xi_{1}\right), \mathcal{U}_{b_{1,2}}\left(\xi_{2}\right)$ and $\mathcal{U}_{b_{1,2} \circ_{1} b_{1,2}}\left(\xi_{1} \otimes \xi_{2}\right)$ vanish unless $\operatorname{deg} \xi_{1}=\operatorname{deg} \xi_{2}=$ 1. So we are left to check that $\mathcal{U}_{b_{1,2}}\left(\xi_{1}\right) \circ_{1} \mathcal{U}_{b_{1,2}}\left(\xi_{2}\right)=\mathcal{U}_{b_{1,2} \circ_{1} b_{1,2}}\left(\xi_{1} \otimes \xi_{2}\right)$ when both $\xi_{1}$ and $\xi_{2}$ are 2 -vector fields. We begin by computing $\mathcal{U}_{b_{1,2}}\left(\xi_{1}\right) \circ_{1} \mathcal{U}_{b_{1,2}}\left(\xi_{2}\right)$ :

$$
\begin{aligned}
\left\langle\mathcal{U}_{b_{1,2}}\left(\xi_{1}\right) \circ_{1}\right. & \mathcal{U}_{b_{1,2}}\left(\xi_{2}\right)|f \otimes g \otimes h\rangle=\left\langle\mathcal{U}_{b_{1,2}}\left(\xi_{1}\right) \mid\left\langle\mathcal{U}_{b_{1,2}}\left(\xi_{2}\right) \mid f \otimes g\right\rangle \otimes h\right\rangle \\
= & \left\langle\mathcal{U}_{b_{1,2}}\left(\xi_{1}\right) \mid 2 \xi_{2}^{i_{3} i_{4}}\left(\partial_{i_{3}} f\right)\left(\partial_{i_{4}} g\right) \otimes h\right\rangle \\
= & 4 \xi_{1}^{i_{1} i_{2}} \partial_{i_{1}}\left(\xi_{2}^{i_{3} i_{4}}\left(\partial_{i_{3}} f\right)\left(\partial_{i_{4}} g\right)\right)\left(\partial_{i_{2}} h\right) \\
= & 4 \xi_{1}^{i_{1} i_{2}}\left(\partial_{i_{1}} \xi_{2}^{i_{3} i_{4}}\right)\left(\partial_{i_{3}} f\right)\left(\partial_{i_{4}} g\right)\left(\partial_{i_{2}} h\right)+4 \xi_{1}^{i_{1} i_{2}} \xi_{2}^{i_{3} i_{4}}\left(\partial_{i_{1}} \partial_{i_{3}} f\right)\left(\partial_{i_{4}} g\right)\left(\partial_{i_{2}} h\right) \\
& \quad+4 \xi_{1}^{i_{1} i_{2}} \xi_{2}^{i_{3} i_{4}}\left(\partial_{i_{3}} f\right)\left(\partial_{i_{1}} \partial_{i_{4}} g\right)\left(\partial_{i_{2}} h\right)
\end{aligned}
$$

Now we turn to the computation of $\mathcal{U}_{b_{1,2} \circ_{1} b_{1,2}}$. We have already computed that

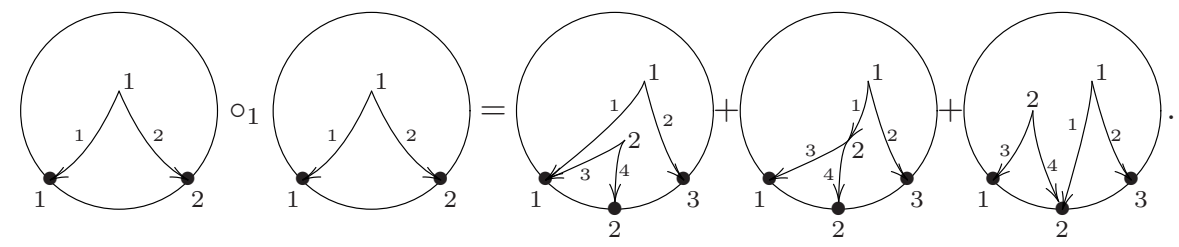

If we denote by $\Phi_{1}, \Phi_{2}$ and $\Phi_{3}$ the three graphs on the right hand side of the above equation, then

$$
\begin{aligned}
\left\langle\mathcal{U}_{\Phi_{1}}\left(\xi_{1} \otimes \xi_{2}\right) \mid f \otimes g \otimes h\right\rangle & =4 \mathcal{U}_{\Phi_{2}}\left(\xi_{1} \otimes \xi_{2}\right)|f \otimes g \otimes h\rangle
\end{aligned}=
$$

and

$$
\begin{aligned}
& \left\langle\mathcal{U}_{\Phi_{3}}\left(\xi_{1} \otimes \xi_{2}\right) \mid f \otimes g \otimes h\right\rangle= \\
& =4 \xi_{1}^{i_{1} i_{2}} \xi_{2}^{i_{3} i_{4}}\left(\partial_{i_{3}} f\right)\left(\partial_{i_{4}} \partial_{i_{1}} g\right)\left(\partial_{i_{2}} h\right)
\end{aligned}
$$


Therefore, $\left\langle\mathcal{U}_{b_{1,2} \circ_{1} b_{1,2}}\left(\xi_{1} \otimes \xi_{2}\right) \mid f \otimes g \otimes h\right\rangle=\left\langle\mathcal{U}_{b_{1,2}} \circ_{1} \mathcal{U}_{b_{1,2}} \mid f \otimes g \otimes h\right\rangle$, as expected.

\section{2 $\mathcal{U}$ as a morphism of complexes}

Lemma 6.2. $d_{1}\left(\mathcal{U}_{\Gamma}\right)=\mathcal{U}_{d_{1} \Gamma}$ and $d_{2}\left(\mathcal{U}_{\Gamma}\right)=\mathcal{U}_{d_{2} \Gamma}$. As a consequence, $d_{C E}\left(\mathcal{U}_{\Gamma}\right)=$ $\mathcal{U}_{d \Gamma}$

Proof. The differential $d_{1}$ is defined as an adjoint both in the Chevalley-Eilenberg complex and in the complex of graphs, and we have already shown that $\mathcal{U}$ is a Lie algebra map $\mathscr{G}^{\bullet}, \bullet \rightarrow C E^{\bullet, \bullet}\left(T_{\text {poly }}^{\bullet}, D_{\text {poly }}^{\bullet}\right)$. Then, the compatibility of $\mathcal{U}$ with the differential $d_{1}$ immediately follows from the fact that $\mathcal{U}$ maps the graph $b_{0,2}$ (inducing $d_{1}$ on the complex of graphs) to the multiplication $\mu$ on $\mathcal{C}^{\infty}\left(\mathbb{R}^{d}\right)$ (inducing $d_{1}$ on the Chevalley-Eilenberg complex).

The compatibility of $\mathcal{U}$ with the differential $d_{2}$ is an immediate consequence of the finer statement $d_{2, i}\left(\mathcal{U}_{\Gamma}\right)=\mathcal{U}_{d_{2, i} \Gamma}$, where $d_{2, i}: \operatorname{Hom}\left(\left(T_{\text {poly }}^{\bullet}\right)^{\otimes q}, D_{\text {poly }}^{\bullet}[p]\right) \rightarrow$ $\operatorname{Hom}\left(\left(T_{\text {poly }}^{\bullet}\right)^{\otimes(q+1)}, D_{\text {poly }}^{\bullet}[p]\right)$ is defined as

$\left(d_{2, i} \mathcal{F}\right)\left(\gamma_{1} \otimes \cdots \otimes \gamma_{i} \otimes \gamma_{i+1} \otimes \cdots \otimes \gamma_{q+1}\right)=\mathcal{F}\left(\gamma_{1} \otimes \cdots \otimes\left(\gamma_{i} \bullet \gamma_{i+1}\right) \otimes \cdots \otimes \gamma_{q+1}\right)$ and $d_{2, i}: \mathscr{G}^{p, q} \rightarrow \mathscr{G}^{p, q+1}$ is the operator which inserts an edge into the $i^{\text {th }}$ vertex of a graph (see also Arn04, AM02]). Therefore, in order to prove the compatibility of $\mathcal{U}$ with the differential $d_{2}$, we have to show that

$$
\mathcal{U}_{d_{2, i} \Gamma}\left(\gamma_{1} \otimes \cdots \otimes \gamma_{i} \otimes \gamma_{i+1} \otimes \cdots \otimes \gamma_{q+1}\right)=\mathcal{U}_{\Gamma}\left(\gamma_{1} \otimes \cdots \otimes\left(\gamma_{i} \bullet \gamma_{i+1}\right) \otimes \cdots \otimes \gamma_{q+1}\right) .
$$

This directly follows from the definition of the Nijenhuis-Richardson pre-Lie operation, when $\gamma_{i}, \gamma_{i+1}$ are two homogeneous polyvector fields. Indeed, if $b_{1, m}$ denotes the $m$-corolla (i.e., the admissible graph with one internal vertex, $m$ boundary vertices and $m$ edges), then the definition of the Nijenhuis-Richardson - operation is equivalent to the following identity:

$$
\mathcal{U}_{d_{2,1} b_{1, m}}\left(\gamma_{1} \otimes \gamma_{2}\right)=\mathcal{U}_{b_{1, m}}\left(\gamma_{1} \bullet \gamma_{2}\right) \text {. }
$$

By linearity, equation (6.1) holds for arbitrary (i.e., possibly non-homogeneous) polyvector fields.

Remark 6.1. Note that, if $\operatorname{deg}\left(\gamma_{j}\right)=k_{j}$, then the evaluation of the terms in the left hand side of equation (6.1) corresponding to the different possible insertions of an oriented edge in the vertex $i$ is non-trivial only when there are exactly $k_{i}+1$ edges stemming from the vertex $i$ and $k_{i+1}+1$ edges stemming from the vertex $i+1$.

Example 6.2. Let $b_{1,2}$ be the 2-corolla. The only nonzero homogeneous component of $\mathcal{U}_{b_{1,2}}: T_{\text {poly }}^{\bullet} \rightarrow D_{\text {poly }}^{\bullet}$ is:

$$
\mathcal{U}_{b_{1,2}}: T_{\text {poly }}^{1} \rightarrow D_{\text {poly }}^{1},
$$

which is the natural identification of 2 -vector fields with 2-derivations. The equations we want to verify are

$$
\left(d_{2,1} \mathcal{U}_{b_{1,2}}\right)\left(\xi_{1} \otimes \xi_{2}\right)=\mathcal{U}_{b_{1,2}}\left(\xi_{1} \bullet \xi_{2}\right)=\mathcal{U}_{d_{2,1} b_{1,2}}\left(\xi_{1} \otimes \xi_{2}\right)
$$


These equations are trivial for homogeneous polyvector fields unless $\operatorname{deg}\left(\xi_{1} \bullet\right.$ $\left.\xi_{2}\right)=1$, i.e. unless $\operatorname{deg} \xi_{1}+\operatorname{deg} \xi_{2}=1$. In these cases the first equation reduces to

$$
\left(d_{2,1} \mathcal{U}_{b_{1,2}}\right)\left(\xi_{1} \otimes \xi_{2}\right)=\xi_{1} \bullet \xi_{2}
$$

Since the degree of a poly-vector field is at least -1 , we have only two possibilities (up to a permutation), namely $\operatorname{deg} \xi_{1}=1, \operatorname{deg} \xi_{2}=0$ or $\operatorname{deg} \xi_{1}=2, \operatorname{deg} \xi_{2}=$ -1 . Let us compute $\xi_{1} \bullet \xi_{2}$ in the first case. We find

$$
\xi_{1} \bullet \xi_{2}=2 \xi_{1}^{i_{1} i_{2}}\left(\partial_{i_{1}} \xi_{2}^{i_{3}}\right) \partial_{i_{2}} \wedge \partial_{i_{3}} .
$$

Therefore,

$$
\begin{aligned}
\left\langle\left(d_{2,1} \mathcal{U}_{b_{1,2}}\right)\left(\xi_{1} \otimes \xi_{2}\right) \mid f \otimes g\right\rangle= & 2 \xi_{1}^{i_{1} i_{2}}\left(\partial_{i_{1}} \xi_{2}^{i_{3}}\right)\left(\partial_{i_{2}} f\right)\left(\partial_{i_{3}} g\right) \\
& -2 \xi_{1}^{i_{1} i_{2}}\left(\partial_{i_{1}} \xi_{2}^{i_{3}}\right)\left(\partial_{i_{3}} f\right)\left(\partial_{i_{2}} g\right)
\end{aligned}
$$

Now, we compute $\left\langle\mathcal{U}_{d_{2,1} b_{1,2}}\left(\xi_{1} \otimes \xi_{2}\right) \mid f \otimes g\right\rangle$. We have already computed

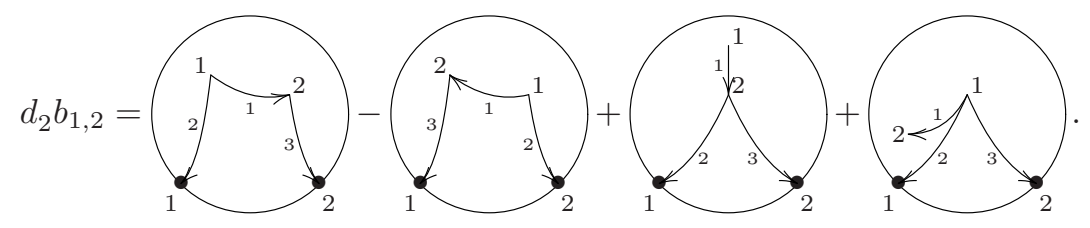

If we denote the 4 graphs on the right hand side by $\Phi_{1}, \ldots, \Phi_{4}$, then the only non-zero pairings are the following:

$$
\begin{aligned}
& \left\langle\mathcal{U}_{\Phi_{1}}\left(\xi_{1} \otimes \xi_{2}\right) \mid f \otimes g\right\rangle={ }_{f}^{i_{2}} \\
& =2 \xi_{1}^{i_{1} i_{2}}\left(\partial_{i_{1}} \xi_{2}^{i_{3}}\right)\left(\partial_{i_{2}} f\right)\left(\partial_{i_{3}} g\right),
\end{aligned}
$$

and

$$
\left\langle\mathcal{U}_{\Phi_{2}}\left(\xi_{1} \otimes \xi_{2}\right) \mid f \otimes g\right\rangle=\underbrace{\xi_{1}^{i_{1} i_{2}}\left(\partial_{i_{1}}\right.}_{f} \xi_{2}^{i_{3}})\left(\partial_{i_{3}} f\right)\left(\partial_{i_{2}} g\right)
$$

Therefore, if $\operatorname{deg} \xi_{1}=1$ and $\operatorname{deg} \xi_{2}=0$, we find

$$
\begin{aligned}
\left\langle\mathcal{U}_{d_{2,1} b_{1,2}}\left(\xi_{1} \otimes \xi_{2}\right) \mid f \otimes g\right\rangle & =\left\langle\mathcal{U}_{\Phi_{1}}\left(\xi_{1} \otimes \xi_{2}\right) \mid f \otimes g\right\rangle-\left\langle\mathcal{U}_{\Phi_{2}}\left(\xi_{1} \otimes \xi_{2}\right) \mid f \otimes g\right\rangle \\
= & 2 \xi_{1}^{i_{1} i_{2}}\left(\partial_{i_{1}} \xi_{2}^{i_{3}}\right)\left(\partial_{i_{2}} f\right)\left(\partial_{i_{3}} g\right) \\
& \quad-2 \xi_{1}^{i_{1} i_{2}}\left(\partial_{i_{1}} \xi_{2}^{i_{3}}\right)\left(\partial_{i_{3}} f\right)\left(\partial_{i_{2}} g\right) \\
= & \left\langle\left(d_{2,1} \mathcal{U}_{b_{1,2}}\right)\left(\xi_{1} \otimes \xi_{2}\right) \mid f \otimes g\right\rangle
\end{aligned}
$$


Now we consider the second possibility, namely $\operatorname{deg} \xi_{1}=2$ and $\operatorname{deg} \xi_{2}=-1$. In this case the Nijenhuis-Richardson bracket is

$$
\xi_{1} \bullet \xi_{2}=3 \xi_{1}^{i_{1} i_{2} i_{3}}\left(\partial_{i_{1}} \xi_{2}\right) \partial_{i_{2}} \wedge \partial_{i_{3}}
$$

Therefore,

$$
\left\langle\left(d_{2,1} \mathcal{U}_{b_{1,2}}\right)\left(\xi_{1} \otimes \xi_{2}\right) \mid f \otimes g\right\rangle=6 \xi_{1}^{i_{1} i_{2} i_{3}}\left(\partial_{i_{1}} \xi_{2}\right)\left(\partial_{i_{2}} f\right)\left(\partial_{i_{3}} g\right)
$$

In computing $\left\langle\mathcal{U}_{d_{2,1} b_{1,2}}\left(\xi_{1} \otimes \xi_{2}\right) \mid f \otimes g\right\rangle$ the only non trivial pairing is

$$
\begin{aligned}
\mathcal{U}_{\Phi_{4}}\left(\xi_{1} \otimes \xi_{2}\right) & = \\
& =6 \xi_{1}^{i_{1} i_{2} i_{3}}\left(\partial_{i_{1}} \xi_{2}\right)\left(\partial_{i_{2}} f\right)\left(\partial_{i_{3}} g\right) \\
& =d_{2,1}\left(\mathcal{U}_{b_{1,2}}\right)\left(\xi_{1} \otimes \xi_{2}\right)|f \otimes g\rangle
\end{aligned}
$$

Therefore, if $\operatorname{deg} \xi_{1}=2$ and $\operatorname{deg} \xi_{2}=-1$, we find

$$
\left\langle\mathcal{U}_{d_{2,1} b_{1,2}}\left(\xi_{1} \otimes \xi_{2}\right) \mid f \otimes g\right\rangle=\left\langle\mathcal{U}_{\Phi_{4}}\left(\xi_{1} \otimes \xi_{2}\right) \mid f \otimes g\right\rangle=\left\langle\left(d_{2,1} \mathcal{U}_{b_{1,2}}\right)\left(\xi_{1} \otimes \xi_{2}\right) \mid f \otimes g\right\rangle
$$

\section{The Maurer-Cartan equation on graphs and the cocycle equation}

We have seen in Section 4 that an $L_{\infty}$-morphism between $T_{\text {poly }}^{\bullet}$ and $D_{\text {poly }}^{\bullet}$ can be viewed as a solution of the vertical Maurer-Cartan equation on $C E^{\bullet}\left(T_{\text {poly }}^{\bullet}, D_{\text {poly }}^{\bullet}\right)$. Therefore, the differential graded Lie algebra morphism $\mathcal{U}$ reduces the problem of writing an $L_{\infty}$-morphism between $T_{\text {poly }}^{\bullet}$ and $D_{\text {poly }}^{\bullet}$ to the problem of solving the vertical Maurer-Cartan equation on the complex of graphs. This will in turn be equivalent to solving the cocycle equation which is the dual of the vertical Maurer-Cartan equation.

\subsection{The dual dg-Lie-coalgebra structure}

We begin by passing from the graded Lie algebra of graphs $\mathscr{G} \bullet$ to the dual Lie-coalgebra $(\mathscr{G} \bullet)^{*}$, where * means "graded dual". Since we want to interpret also elements in the Lie-coalgebra as graphs, we introduce a symmetric nondegenerate pairing

$$
\langle\mid\rangle: \mathscr{G} \bullet \bullet \bullet \mathscr{G} \bullet \bullet \bullet \mathbb{C}
$$

by declaring the set $\mathcal{G}^{\bullet}, \bullet$ of isomorphism classes of admissible graphs to be an orthonormal basis of $\mathscr{G}^{\bullet \bullet} \bullet$. This means that we are identifying $\left(\mathscr{G}^{p, q}\right)^{*}$ with $\mathscr{G}^{p, q}$. The pairing $\langle\mid\rangle$ induces a differential graded Lie coalgebra structure on $\mathscr{G} \bullet \bullet \bullet$ 
which we will now describe explicitly. The codifferential $d_{2}^{*}: \mathscr{G}^{p, q} \rightarrow \mathscr{G}^{p, q-1}$ is defined by the equation

$$
\left\langle d_{2}^{*} \Phi \mid \Gamma\right\rangle=\left\langle\Phi \mid d_{2} \Gamma\right\rangle
$$

for any $\Phi, \Gamma$. Therefore

$$
\begin{aligned}
d_{2}^{*} \Phi & =\sum_{\Gamma}\left\langle d_{2}^{*} \Phi \mid \Gamma\right\rangle \Gamma=\sum_{\Gamma}\left\langle\Phi \mid d_{2} \Gamma\right\rangle \Gamma \\
& =\sum_{\Gamma} \sum_{\substack{e \hookrightarrow \Psi \rightarrow \Gamma \\
\text { internal }}} \pm\langle\Phi \mid \Psi\rangle \Gamma=\sum_{\substack{e \leftrightarrow \Phi \rightarrow \Gamma \\
\text { internal }}} \pm \Gamma \\
& =\sum_{\substack{e \in \text { Edges }(\Phi) \\
\text { internal }}} \pm \Phi / e
\end{aligned}
$$

i.e., $d_{2}^{*} \Phi$ is the sum over all the internal edge contractions of $\Phi$. Note that this is the Kontsevich graph homology differential Kon92, p.109 ([Kon93, p.175). To determine an explicit expression for the Lie co-bracket

$$
\delta: \mathscr{G}^{p, q} \rightarrow \bigoplus_{\substack{p_{1}+p_{2}=p \\ q_{1}+q_{2}=q}} \mathscr{G}^{p_{1}, q_{1}} \otimes \mathscr{G}^{p_{2}, q_{2}}
$$

we argue in a similar way:

$$
\langle\delta \Phi \mid \Gamma \otimes \Psi\rangle=\langle\Phi \mid[\Gamma, \Psi]\rangle
$$

so that

$$
\begin{aligned}
\delta \Phi & =\sum_{\Gamma, \Psi}\langle\delta \Phi \mid \Gamma \otimes \Psi\rangle \Gamma \otimes \Psi=\sum_{\Gamma, \Psi}\langle\Phi \mid[\Gamma, \Psi]\rangle \Gamma \otimes \Psi \\
& =\sum_{\Gamma, \Psi} \sum_{\substack{\Gamma \rightarrow \Xi \rightarrow \Psi \\
\text { boundary }}} \pm\langle\Phi \mid \Xi\rangle(\Gamma \otimes \Psi \pm \Psi \otimes \Gamma) \\
& =\sum_{\substack{\Gamma \varangle \Phi \\
\text { boundary }}} \pm(\Gamma \otimes \Phi / \Gamma \pm \Phi / \Gamma \otimes \Gamma) .
\end{aligned}
$$

Note that the Lie cobracket $\delta$ coincides with the anti-symmetrization of the coproduct $\Delta$ introduced by Ionescu in [on03] (see also [on04b, Ion04c]).

\subsection{The Maurer-Cartan equation on graphs and the cocy- cle equation}

In this section we will start our study of the solutions of the vertical MaurerCartan equation

$$
d_{2} \gamma+\frac{1}{2}[\gamma, \gamma]=0
$$

in the Hochschild-Kontsevich differential Lie algebra of graphs. 
Thanks to the non-degenerate pairing $\langle\mid\rangle$, the above equation is equivalent to

$$
\left\langle d_{2} \gamma+\frac{1}{2}[\gamma, \gamma] \mid \Phi\right\rangle=0, \quad \forall \Phi \in \mathcal{G}^{2}
$$

Now, we use the fact that $\mathcal{G}^{1}$ is a linear basis of $\mathscr{G}^{1}$ to write

$$
\gamma=\sum_{\Gamma \in \mathcal{G}^{1}} W_{\Gamma} \Gamma
$$

for some constants $W_{\Gamma}$. The Maurer-Cartan equation then becomes

$$
\sum_{\Gamma} W_{\Gamma}\left\langle d_{2} \Gamma \mid \Phi\right\rangle+\frac{1}{2} \sum_{\Gamma_{1}, \Gamma_{2}} W_{\Gamma_{1}} W_{\Gamma_{2}}\left\langle\left[\Gamma_{1}, \Gamma_{2}\right] \mid \Phi\right\rangle=0, \quad \forall \Phi \in \mathcal{G}^{2}
$$

i.e.

$$
\sum_{\Gamma} W_{\Gamma}\left\langle\Gamma \mid d_{2}^{*} \Phi\right\rangle+\frac{1}{2} \sum_{\Gamma_{1}, \Gamma_{2}} W_{\Gamma_{1}} W_{\Gamma_{2}}\left\langle\Gamma_{1} \otimes \Gamma_{2} \mid \delta \Phi\right\rangle=0, \quad \forall \Phi \in \mathcal{G}^{2}
$$

The map $W: \Gamma \mapsto W_{\Gamma}$ can be seen as a linear functional on $\mathscr{G}^{1}$, i.e., as an element of the space of "weights". Having this in mind, the above equation can be rewritten as the cocycle equation

$$
W\left(d_{2}^{*} \Phi\right)+\frac{1}{2} W^{\otimes 2}(\delta \Phi)=0, \quad \forall \Phi \in \mathcal{G}^{2}
$$

which explicitly reads

$$
\sum_{\substack{e \in E_{\Phi} \\ \text { internal }}} \pm W_{\Phi / e}+\sum_{\substack{\Gamma \triangleleft \Phi \\ \text { boundary }}} \pm W_{\Gamma} W_{\Phi / \Gamma}=0, \quad \forall \Phi \in \mathcal{G}^{2}
$$

We will call any solution $W$ of the above equation a cocycle. The reason for this is that $W$ is actually a cocycle for the cobar differential corresponding to the coalgebra structure on $\mathscr{G} \mathscr{\bullet}^{\bullet}$; see details in subsection $\mathbf{7 . 4}$

Remark 7.1. In order to make direct contact with the original proof by Kontsevich in Kon97, we introduced the somehow artificial self-duality of the space of graphs by imposing that graphs form an orthonormal basis. Actually, the natural dual of the space of graphs is the space of weights, and the cocycle equation should be directly read as the dual Maurer-Cartan equation for the dg-Lie-coalgebra structure on the space of weights.

Summing up what we have proved so far in the paper, we obtain:

Proposition 7.1. Let $W$ be any cocycle such that $W_{b_{0,2}}=1$. Then $\mathcal{F}^{\mu}=$ $\sum_{\Gamma \in \mathcal{G}^{1}} W_{\Gamma} \mathcal{U}_{\Gamma}$ is an $L_{\infty}$-morphism between $T_{\text {poly }}^{\bullet}$ and $D_{\text {poly }}^{\bullet}$. 
Remark 7.2. As seen in Remark 4.1 we obtain a deformation quantization of Poisson structures out of any $\mathcal{F}^{\mu}$ such that $\mathcal{F}_{1}^{\mu}: T_{\text {poly }}^{m} \rightarrow D_{\text {poly }}^{m}$ is the canonical identification of polyvector fields with polydifferential operators. Since the restriction of $\sum_{\Gamma \in \mathcal{G}^{1}} W_{\Gamma} \mathcal{U}_{\Gamma}$ to $\operatorname{Hom}\left(T_{\text {poly }}^{m-1}, D_{\text {poly }}^{m-1}\right)$ is $W_{b_{1, m}} \mathcal{U}_{b_{1, m}}$ and $\mathcal{U}_{b_{1, m}}(\xi)=m ! \xi$, we get a star-product quantization of Poisson structures out of any cocycle with $W_{b_{1, m}}=\frac{1}{m !}$. The formula for the star-product is

$$
f \star_{\alpha} g=\sum_{\Gamma \in \mathcal{G}^{1}} W_{\Gamma} \mathcal{U}_{\Gamma}(\exp \{\hbar \alpha\})(f, g)=\sum_{q=0}^{\infty} \sum_{\Gamma \in \mathcal{G}^{1-q, q}} \frac{\hbar^{q}}{q !} W_{\Gamma} \mathcal{U}_{\Gamma}\left(\alpha^{\wedge q}\right)(f, g) .
$$

Recall from Section 2 that $\mathcal{G}^{1-q, q}$ is the set of isomorphism classes of all admissible graphs $\Gamma$ with $q$ internal vertices and such that $e(\Gamma)-v(\Gamma)+2=q$. Here $v(\Gamma)$ and $e(\Gamma)$ are the number of vertices and edges of $\Gamma$, respectively. So, if we denote by $n$ the number of internal vertices and by $m$ the number of boundary vertices of $\Gamma$, a graph in $\mathcal{G}^{1-q, q}$ satisfies $n=q$ and $e(\Gamma)=2 n+m-2$. Since $\alpha$ is a 2-vector field, the admissible graphs actually appearing in the star-product formula must have exactly two outgoing edges for any internal vertex, and we get the identity

$$
2 n=e(\Gamma)=2 n+m-2
$$

which implies $m=2$, as it should be since $\mathcal{U}_{\Gamma}$ must be an element of $D_{\text {poly }}^{1}$. Then, if we denote by $\mathcal{G}_{n, 2}^{1}$ the set of isomorphism classes of degree one admissible graphs with $n$ internal and two boundary vertices, and for any graph $\Gamma$ in $\mathcal{G}_{n, 2}^{1}$ we write $B_{\Gamma, \alpha}$ for $\mathcal{U}_{\Gamma}\left(\alpha^{\wedge n}\right)$, the formula for the star-product gets the familiar aspect (see [Kon97])

$$
f \star_{\alpha} g=\sum_{n=0}^{\infty} \frac{\hbar^{n}}{n !} \sum_{\Gamma \in \mathcal{G}_{n, 2}^{1}} W_{\Gamma} B_{\Gamma, \alpha}(f, g) .
$$

The problem of (local) deformation quantization is thus reduced to solving the cocycle equation on graphs, i.e., to determining a system of weights $W_{\Gamma}$ satisfying equation (7.2). Such a system of weights has first been found in Kon97, where the $W_{\Gamma}$ are defined as integrals on suitable configuration spaces of points in a disk, naturally associated to admissible graphs. For a sigma model/quantum field theory interpretation of these configuration space integrals, we refer the reader to CF99, Ion03. An attempt towards a combinatorial solution of the cocycle equation can be found in Ion04a. 


\subsection{Connes-Kreimer convolution and Kontsevich solution}

The cocycle equation can be seen as a Connes-Kreimer convolution with some vanishing coefficients. Namely, equation (7.2) can be rewritten as

$$
\begin{cases}\sum_{\begin{array}{c}
\Gamma \text { proper } \\
\text { subgraph of } \Phi
\end{array}} W_{\Gamma} W_{\Phi / \Gamma}=0, \quad \forall \Phi \in \mathcal{G}^{2} \\
W_{\Gamma} W_{\Phi / \Gamma}=0, \quad \text { unless } \Gamma \text { is an internal edge or } \\
\Gamma \text { is a boundary normal subraph. }\end{cases}
$$

The left-hand side of the first equation in (7.3) is Connes-Kreimer convolution $W \star W$, and the equation $W \star W=0$ should be thought as an abstract version of the Stokes formula on configuration spaces. Indeed, if Conf denotes the configuration space functor mapping an admissible graph $\Gamma$ to the configuration space of the vertices of $\Gamma$ into a manifold with boundary (in the interior or on the boundary of the manifold depending on the type of the vertices), $\omega_{\Gamma}$ denotes an angle form induced by an embedding of $\Gamma$, and $\mathbb{H}$ denotes the Poincaré disk, then one can define the weights as $W=\langle\overline{\operatorname{Conf}}(\mathbb{H}) \mid \omega\rangle$, i.e., $W_{\Gamma}=\int_{\overline{\operatorname{Conf}}_{\Gamma}(\mathbb{H})} \omega_{\Gamma}$. Then,

$$
\begin{array}{r}
W \star W=\langle\overline{\operatorname{Conf}}(\mathbb{H}) \otimes \overline{\operatorname{Conf}}(\mathbb{H}) \mid \omega \wedge \omega\rangle \\
\quad=\langle\partial \overline{\operatorname{Conf}}(\mathbb{H}) \mid \omega\rangle=\langle\overline{\operatorname{Conf}}(\mathbb{H}) \mid d \omega\rangle=0,
\end{array}
$$

where the last equality follows from the fact that angle forms are closed: $d \omega=0$. This shows that the first equation in (7.3) is automatically satisfied as soon as one defines the weights as configuration space integrals. The proof of the cocycle equation is then reduced to the checking of the vanishing of the configuration space integrals corresponding to the terms in the second line of (7.3), as in Kon97. See the Appendix for details on configuration space integrals in Kontsevich's proof.

\subsection{Configuration spaces and the cobar construction}

We now explain in which sense a solution $W$ to the cocycle equation (7.2) is a cocycle. Recall Qui69 that a DGLA structure on a vector space $\mathfrak{g}$ can be seen as a codifferential $Q$ which is a coderivation on $C(\mathfrak{g}[1])=\bigoplus_{n \geq 1} S^{n}(\mathfrak{g}[1])$, the cofree coassociative cocommutative coalgebra without counit cogenerated by $\mathfrak{g}[1]$. In our case, $\mathfrak{g}=\mathscr{G} \bullet$ and the codifferential $Q$ is $d_{2}^{*}+\delta$. We have remarked that the cobraket $\delta$ is actually the antisymmetrization of a coproduct $\Delta$, which is dual to the pre-Lie operation $\circ$. This implies Ion03 that the differential $d_{2}^{*}+\delta$ on $C(\mathscr{G} \bullet[1])$ is induced by the differential $D=d_{2}^{*}+\Delta$ on $T_{+}(\mathscr{G} \bullet[1])=\bigoplus_{n>1} T^{n}(\mathscr{G} \bullet[1])$, the cofree coassociative coalgebra without counit cogenerated by $\mathscr{G} \bullet[1]$. Note that $T_{+}(\mathscr{G} \bullet[1])$ is nothing else than the graded vector space underlying the cobar complex $F\left(\mathscr{G}^{\bullet}\right)$ of $\mathscr{G} \bullet$ (see [Jon02, p.111). 
The differential $D$ can be seen as a perturbation of the differential $d_{2}^{*}$ of the cobar complex. Note that $D=d_{2}^{*}+\Delta$ is the differential yielding the cohomology of Feynman-Kontsevich graphs Ion03 Ion04b Ion04c]. The system of weights $W$ is as linear functional on $T_{+}(\mathscr{G} \bullet[1])$; from this point of view, the cocycle equation is nothing but the equation ${ }^{t} D W=0$, where ${ }^{t} D$ is the dual differential on $\operatorname{Hom}\left(T_{+}(\mathscr{G} \bullet[1]) ; \mathbb{C}\right)$. In other words, $W$ is a ${ }^{t} D$-cocycle.

The operator $D$ provides a nice interpretation of the fact that Kontsevich's weights do indeed provide a cocycle. Recall from the previous subsection that $W_{\Gamma}$ is defined using a rule $\omega$ which maps an admissible graph $\Gamma$ to a differential form $\omega_{\Gamma}$ on the configuration space of the vertices of $\Gamma$ in the Poincaré disk:

$$
\omega: \mathscr{G} \bullet \rightarrow \Omega^{\bullet}(\overline{\operatorname{Conf}}(\mathbb{H}) ; \mathbb{R})
$$

Such a form is the angle form; for details, in addition to Kon97, see Pol02. The map $\omega$ can be extended to a map between the cobar constructions on graphs and on the space of differential forms on configuration spaces. Using the familiar fact that differential forms on a product are the tensor product of differential forms on the factors, we get a map

$$
\omega:\left\{T_{+}(\mathscr{G} \bullet[1]), D\right\} \rightarrow\left\{\Omega^{\bullet}\left(T_{+}(\overline{\operatorname{Conf}}(\mathbb{H})) ; \mathbb{R}\right), d\right\},
$$

which is a map of complexes. ${ }^{2}$ The weights $W$ on the cobar complex of graphs are then defined as $W=\left\langle T_{+}(\overline{\operatorname{Conf}}(\mathbb{H})) \mid \omega\right\rangle$, where the angle form corresponding to a tensor product of admissible graphs is integrated over the corresponding component in $T_{+}(\overline{\operatorname{Conf}}(\mathbb{H}))$. The proof that Kontsevich coefficients yield a cocycle then reads as follows: for any admissible graph $\Gamma$,

$$
\left\langle{ }^{t} D W \mid \Gamma\right\rangle=\langle W \mid D \Gamma\rangle=\left\langle T_{+}(\overline{\operatorname{Conf}}(\mathbb{H})) \mid \omega_{D \Gamma}\right\rangle=\left\langle T_{+}(\overline{\operatorname{Conf}}(\mathbb{H})) \mid d \omega_{\Gamma}\right\rangle=0,
$$

since angle forms are closed.

\section{The tree-level approximation}

In the main body of the paper we have reviewed how an $L_{\infty}$-morphism between $T_{\text {poly }}^{\bullet}$ and $D_{\text {poly }}^{\bullet}$ can be written as a sum over admissible graphs, leading to a deformation quantization of Poisson structures. We now show how the DGLA point of view we adopted provides a nice algebraic setting to discuss the tree-level approximation of Kontsevich's star-product. Namely, we discuss the possibility of writing a star-product formula as a sum over the family of Kontsevich's forests, i.e. admissible graphs whose underlying non-oriented graphs become acyclic ${ }^{3}$ when one removes the boundary vertices. There are indeed several hints that such a formula may exist. For instance, constant Poisson structures admit the Moyal's star-product quantization which can be seen as a

\footnotetext{
${ }^{2}$ This is, in a slightly different formulation from the original paper, Theorem 3.2 of Ion03.

${ }^{3} \mathrm{~A}$ non-oriented graph is called acyclic when all of its connected components are simply connected.
} 
sum over graphs with no edge ending on an internal vertex, and Michael Polyak has shown in Pol02 that the Kontsevich's star-product for linear Poisson structures is gauge-equivalent to the Gutt's star-product, which can be seen as a sum over the family of Kontsevich's forests. ${ }^{4}$ It should be remarked that Polyak's proof relies on the linearity of the coefficients of the Poisson structures and so does not provide an argument to decide whether the family of forests could be universal, in the sense that a formula written as a sum over forests could work for any Poisson structure. Additional evidence towards the existence of such a universal formula is provided in Ion04a, where the cocycle condition is investigated from a combinatorial point of view (see also IS05).

In this final section we sketch how an analysis of the tree-level approximation can be obtained by looking at the cohomology of the complex of admissible graphs. The first thing we note is that Kontsevich's forests span a subcomplex $\left(\mathscr{F}^{\bullet}, d_{2}\right)$ of $\left(\mathscr{G}^{\bullet}, d_{2}\right)$, since inserting an internal edge does not change the homotopy type of a graph. On the other hand, $\mathscr{F} \bullet$ is not a DGLA, since the Lie bracket of two forest graphs in not necessarly a forest, as the following example shows

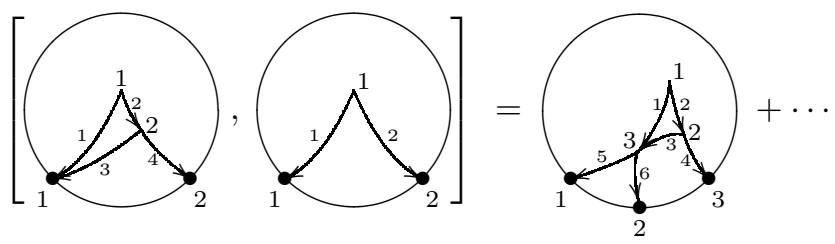

Therefore we cannot immediately reduce the tree-level approximation to a cocycle equation for forests. But one can show (see for instance Kon99) that the inclusion $\iota: \mathscr{F} \bullet \hookrightarrow \mathscr{G} \bullet$ is a quasi-isomorphism of complexes. Then, by homotopical transfer of structure (see KS00, Mar99]) $\mathscr{F} \bullet$ inherits from the DGLA structure of admissible graphs an $L_{\infty}$-structure making $\mathscr{F} \bullet$ and $\mathscr{G} \bullet$ homotopy equivalent $L_{\infty}$-algebras, via an $L_{\infty}$-morphism $\iota_{\infty}: \mathscr{F} \bullet \rightarrow \mathscr{G}^{\bullet}$. The homotopy equivalence $\iota_{\infty}$ is a non-linear map, ${ }^{5}$ whose linear term coincides with the inclusion $\iota$. By Kontsevich-Schlessinger deformation theory Kon97] the solutions of the Maurer-Cartan equation on the DGLA $\mathscr{G} \bullet$ bijectively correspond (up to gauge equivalence) to solutions of the Maurer-Cartan equation $\sum_{n=1}^{\infty}\left[\gamma^{\wedge n}\right]_{n} / n !=0$ on the $L_{\infty}$-algebra $\mathscr{F} \bullet$. In particular, Kontsevich's solution on $\mathscr{G}^{\bullet}$ will induce a solution $\gamma_{\infty}=\sum_{T \in \mathcal{F}^{1}} W_{\infty, T} T$, which is a sum over forest graphs. Note that, due to the non-linearity of the homotopical isomophism $\iota_{\infty}$, the weights $W_{\infty}$ are not simply the restriction of the weights $W$ to forest graphs. To relate the sum over forests $\gamma_{\infty}$ to the tree-level approximation of Kontsevich's star-product, we compose the homotopical isomorphism $\iota_{\infty}$ with the DGLA map $\mathcal{U}$, to get an $L_{\infty}$-morphism $\mathcal{U}_{\infty}: \mathscr{F}^{\bullet} \rightarrow C E^{\bullet}\left(T_{\text {poly }}^{\bullet}, D_{\text {poly }}^{\bullet}\right)$, whose linear part $\mathcal{U}_{\infty, 1}$ is just the restriction of $\mathcal{U}$ to forest graphs. By construction, evaluating $\mathcal{U}_{\infty}$ on $\gamma_{\infty}$ is the same thing as evaluating $\mathcal{U}$ on the Kontsevich's solution, so

\footnotetext{
${ }^{4}$ The reader interested in Kontsevich's star-product for linear Poisson structures is also addressed to ABM99 and Dit99.

${ }^{5}$ Note that, since $\mathscr{F} \bullet$ is not closed under the Lie bracket, the quadratic component $\iota_{\infty, 2}$ is non-trivial.
} 
we have found an expression for the formality $L_{\infty}$-morphism between $T_{\text {poly }}^{\bullet}$ and $D_{\text {poly }}^{\bullet}$ which is a sum over forest graphs. Explicitly,

$$
\begin{aligned}
\mathcal{U}_{\infty}\left(\gamma_{\infty}\right) & =\sum_{n=1}^{\infty} \frac{1}{n !} \mathcal{U}_{\infty, n}\left(\gamma_{\infty}^{\wedge n}\right) \\
& =\sum_{T \in \mathcal{F}^{1}} W_{\infty, T} \mathcal{U}_{T}+\frac{1}{2} \sum_{T_{1}, T_{2} \in \mathcal{F}^{1}} W_{\infty, T_{1}} W_{\infty, T_{2}} \mathcal{U}_{\infty, 2, T_{1}, T_{2}}+\cdots
\end{aligned}
$$

Evaluating this expression on $\exp \{\hbar \alpha\}$, for a given Poisson bivector field $\alpha$, one finds

Theorem 8.1. The Kontsevich's star-product formula quantizing a Poisson structure $\alpha$ on $\mathbb{R}^{d}$ can be rewritten as

$$
\begin{aligned}
f \star_{\alpha} g=\underbrace{\sum_{n=0}^{\infty} \frac{\hbar^{n}}{n !} \sum_{T \in \mathcal{F}_{n, 2}^{1}} W_{\infty, T} B_{T, \alpha}(f, g)}_{\text {tree level approximation }}+ \\
+\underbrace{\sum_{k=2}^{\infty} \frac{1}{k !} \sum_{n=0}^{\infty} \hbar^{n} \sum_{T_{i} \in \mathcal{F}_{m_{i}, n_{i}}^{1}}\left(\prod_{i=1}^{k} \frac{W_{\infty, T_{i}}}{n_{i} !}\right) B_{\infty, T_{1}, \ldots, T_{k}, \alpha}(f, g)}_{\text {correction terms coming from loops }},
\end{aligned}
$$

where the sum ranges over the $n_{i}$ and $m_{j}$ such that $n_{1}+\cdots+n_{k}=n$ and $m_{1}+\cdots+m_{k}=2+k-1$.

In more colloquial terms, the theorem above states that it is possible to write a star-product formula as a sum over forest graphs; this formula is given by a Kontsevich-type formula restricted to forest graphs (but with different weights), the so-called tree-level or semi-classical approximation, plus corrections corresponding to contributions coming from non simply-connected graphs in the original Kontsevich's formula. In the sum-over-forests reformulation, these contributions are written in terms of the multilinear operations $T_{1} \wedge \cdots \wedge T_{k} \mapsto$ $B_{\infty, T_{1}, T_{2}, \ldots, T_{k}, \alpha}$ on forest graphs.

It should be remarked that, since the tree-level approximation depends on the choice of the homotopy equivalence $\iota_{\infty}$, it is far from being unique. Thus we have several different tree-level approximations of Kontsevich's formula, which correspond to different systems of weights $W_{\infty}$; clearly the various tree-level approximations are gauge-equivalent to each other. Recasted into these terms, Polyak's result mentioned at the beginning of this section states that for a linear Poisson structure $\alpha$ there exist a choice of $\iota_{\infty}$ (i.e., a choice of the weights $\left.W_{\infty}\right)$ such that the tree-level approximation is exact. The problem of deciding whether for an arbitrary Poisson structure $\alpha$ there exist an exact tree-level approximation remains open. Yet, it is not unlikely that a more refined study of the $L_{\infty}$-algebra structure on $\mathscr{F} \bullet$, of the multilinear operations $\iota_{\infty, k}: \wedge^{k} \mathscr{F} \bullet \rightarrow$ $\mathscr{G} \bullet[1-k]$ and, consequently, of the multilinear operations $\mathcal{U}_{\infty, k}$ and $B_{\infty, k, \alpha}$ 
on forest graphs may turn to be useful to get a better understanding of this problem. Also it would be interesting to have an interpretation of the weights $W_{\infty}$ in terms of the geometry of suitable configuration spaces.

\section{Appendix: Configuration spaces and Kontsevich's cocycle}

In this section we review Kontsevich's solution of the cocycle equation, yielding the weights $W_{\Gamma}$ in the graph expansion of the formality $L_{\infty}$-morphism. We will not be interested into the physical interpretation behind this construction (see CF99 Ion03 for details); rather we will stress the rôle of the dgLie-coalgebra structure and its relations with Stokes' theorem for configuration spaces of points in a disk, as a crucial ingredient of Kontsevich's proof.

\section{A.1 Configuration spaces and differential forms associated with graphs}

Let $\mathcal{G}_{n, m}$ be the set of (isomorphism classes of) admissible graphs with $n$ internal and $m$ boundary vertices. With any $\Gamma \in \mathcal{G}_{n, m}$, Kontsevich associates a manifold with corners $\bar{C}_{n, m}$ and a closed differential form $\omega_{\Gamma}$ on $\bar{C}_{n, m}$. As the notation suggests, the manifold $\bar{C}_{n, m}$ depends only on the number and type of vertices of $\Gamma$. Indeed, the manifold $\bar{C}_{n, m}$ is the Fulton-MacPherson compactification of the configuration space of $n+m$ points in the disk

$$
\Delta=\{z \in \mathbb{C} \text { such that }\|z\| \leq 1\},
$$

with $n$ points in the interior of the disk and $m$ points on the boundary. Under the usual identification of the boundary of the disk with $\mathbb{R} \cup \infty$, one can assume that the boundary points are represented by $0=t_{1}<t_{2}<\cdots<t_{m}=1$. This means that we are actually fixing an additional point " $\infty$ " on the boundary of the disk. Since the group of complex automorphisms of $\Delta$ fixing $\infty$ has real dimension 2, the real dimension of $\bar{C}_{n, m}$ is $2 n+m-2$. The differential form $\omega_{\Gamma}$ is defined by the following construction: a point of $\bar{C}_{n, m}$ determines a configuration of points in $\Delta$. Use this configuration to draw a copy of $\Gamma$ in the disk, drawing each edge of $\Gamma$ as a geodesic segment (in the usual hyperbolic metric of $\Delta$ ). Then each edge $e$ of $\Gamma$ determines an angle $\phi_{e}$, which is the angle between the edge $e$ and the geodesic joining the starting point of $e$ with the point $\infty$ on the boundary of the disk. As usual, $\phi_{e}$ is locally well defined up to an integral multiple of $2 \pi$ so that the 1 -form $d \phi_{e}$ is well defined. The closed form $\omega_{\Gamma}$ is then defined as the normalized wedge product of all the 1-forms $d \phi_{e}$ with $e$ ranging in the set $E_{\Gamma}$ of edges of $\Gamma$ :

$$
\omega_{\Gamma}=\frac{1}{(2 \pi)^{\left|E_{\Gamma}\right|}} \bigwedge_{e \in E_{\Gamma}} d \phi_{e} .
$$


If $\Gamma$ has bidegree $(p, q)$ then $\left|E_{\Gamma}\right|=2 n+m-(p+q)-1=\operatorname{dim} \bar{C}_{n, m}+1-(p+q)$ and the differential form $\omega_{\Gamma}$ is a top-dimensional differential form on $\bar{C}_{n, m}$ precisely when $\Gamma$ has total degree 1 (see Remark (7.2). Having a manifold and a top-dimensional differential form on it, we can get a real number out of them: define the weight $W_{\Gamma}$, for an admissible graph of total degree 1 , as

$$
W_{\Gamma}=\int_{\bar{C}_{n, m}} \omega_{\Gamma}
$$

On the other hand, if $\Gamma$ has total degree 2 , then the number of edges of $\Gamma$ is $2 n+m-3$, so that we get a top-form on $\bar{C}_{n, m}$ by taking the differential $d \omega_{\Gamma}$, in this case. Stokes' theorem holds for manifold with corners so we get, for any admissible graphs with $n$ internal and $m$ boundary vertices having total degree 2 , the identity

$$
\int_{\partial \bar{C}_{n, m}} \omega_{\Gamma}=\int_{\bar{C}_{n, m}} d \omega_{\Gamma}=0 .
$$

In the next section we will relate the boundary integral on the left to the weights $W_{\Gamma}$ of degree 1 admissible graphs. To do this we need a description of the codimension one boundary components of $\bar{C}_{n, m}$.

\section{A.2 Normal subgraphs and the boundary behavior of the $\omega_{\Gamma}^{\prime}$ 's}

Boundary components of $\bar{C}_{n, m}$ describe the various ways in which a subset of points in a given configuration can collapse to a single point. Since we are dealing with configuration spaces of a manifold with boundary, there are two kinds of degenerations (and so two kinds of boundary components). Degenerations of the first kind are those in which a set $S$ consisting of $n_{1} \geq 2$ internal points collapse to an internal point. In this case, the collapsing makes up a "bubble" and in the limit we are left with a copy of $\mathbb{P}^{1}(\mathbb{C})$ with $n_{1}$ points on it, joined to a disk with $n-n_{1}$ internal points left on it. The joining point of the sphere and the disk is an additional marked point for both spaces. Moreover, the direction pointing towards $\infty$ is a distinguished direction in the disk, and leads to a distinguished tangent vector at $\infty$ on $\mathbb{P}^{1}(\mathbb{C})$. So we get the boundary component

$$
\partial_{S} \bar{C}_{n, m} \simeq \bar{C}_{n_{1}} \times \bar{C}_{n-n_{1}+1, m}
$$

where $\bar{C}_{n_{1}}$ denotes the configuration space of $n_{1}$ points distinct from $\infty$ on $\mathbb{P}^{1}(\mathbb{C})$, up to projective transformations fixing a tangent direction at $\infty$. Note that, since the group of these projective transformations has real dimension 3 , the manifold $\bar{C}_{n_{1}}$ has real dimension $2 n_{1}-3$, and

$$
\operatorname{dim}\left(\partial_{S} \bar{C}_{n, m}\right)=\operatorname{dim}\left(\bar{C}_{n, m}\right)-1 .
$$

Degenerations of the second kind are those in which a set $S$ consisting of $n_{1}$ internal points and a non-empty set $S^{\prime}$ consisting of $m_{1}$ boundary points, with 
$2 n_{1}+m_{1} \geq 2$ collapse to a boundary point. In this case, the collapse produces a new disk joined to the original one by a point in the boundary, which is the $\infty$ point in the new disk. This way we get the boundary component $\partial_{S, S^{\prime}} \bar{C}_{n, m} \simeq$ $\bar{C}_{n_{1}, m_{1}} \times \bar{C}_{n-n_{1}, m-m_{1}+1}$. Note that also in this case we find

$$
\operatorname{dim}\left(\partial_{S, S^{\prime}} \bar{C}_{n, m}\right)=\operatorname{dim}\left(\bar{C}_{n, m}\right)-1 .
$$

Remark A.1. Since it may be a source of confusion, we explicitly note that $\bar{C}_{n, 0}$ and $\bar{C}_{n}$ are different spaces (even their dimensions are different).

Note that the above description of the boundary of $\bar{C}_{n, m}$ produced an asymmetry: there are factors in the boundary components which are associated to admissible graphs (namely, the factors of the form $\bar{C}_{n^{\prime}, m^{\prime}}$ ) and factors which are not associated to graphs (namely, the factors of the form $\bar{C}_{n^{\prime}}$ ). To remedy this asymmetry, we add to admissible graphs a copy of $\mathcal{G}_{n, 0}$, which we will denote by $\mathcal{G}_{n}$. The difference between graphs in $\mathcal{G}_{n}$ and graphs in $\mathcal{G}_{n, 0}$ is that the former have to be thought as embedded into $\mathbb{P}^{1}(\mathbb{C})$, which has no boundary, whereas the latter have to be thought as embedded into $\Delta$, which has a boundary, but the graphs have no boundary vertices. To a graph $\Gamma$ in $\mathcal{G}_{n}$ we will associate the configuration space $\bar{C}_{n}$ and a differential form $\omega_{\Gamma}$ on $\bar{C}_{n}$ by the same rule as before. Note that, in contrast with what happened for $\bar{C}_{n, m}$, the differential form $\omega_{\Gamma}$ will be top-dimensional on $\bar{C}_{n}$ precisely when $\Gamma$ has total degree 2 .

Each subgraph $\Phi$ of $\Gamma$ determines a subset of points, the vertices of $\Phi$, denoted by $v(\Phi)$. Conversely, each subset $S$ (or $S, S^{\prime}$ ) of the vertices of $\Gamma$ determines a full subgraph of $\Gamma$ by the rule "edges of $\Phi$ are those edges of $\Gamma$ which join the vertices in $S$ (or in $S \cup S^{\prime}$ )". The subgraphs corresponding to the sets $S$ and $S, S^{\prime}$ will be denoted $\Gamma_{S}$ and $\Gamma_{S, S^{\prime}}$ respectively.

The boundary behavior of the differential forms $\omega_{\Gamma}$ can be described in a clear way by means of the notion of graph extension introduced in Section [5. Consider a graph $\Gamma$ in $\mathcal{G}_{n, m}$ with total degree 2, and let $S$ be a subset of its internal vertices, with $|S| \geq 2$. Corresponding to $S$ we have a boundary component

$$
\iota_{S}: \bar{C}_{|S|} \times \bar{C}_{n-|S|+1, m} \hookrightarrow \bar{C}_{n, m} .
$$

In addition, we have the subgraph $\Gamma_{S}$ of $\Gamma$ and the quotient graph $\Gamma / \Gamma_{S}$. Assume that $\Gamma_{S}$ is a normal subgraph of $\Gamma$. Then $\Gamma_{S} \in \mathcal{G}_{|S|}$ and $\Gamma / \Gamma_{S} \in \mathcal{G}_{n-|S|+1, m}$. Therefore we have a differential form $\omega_{\Gamma_{S}} \wedge \omega_{\Gamma / \Gamma_{S}}$ on $\bar{C}_{|S|} \times \bar{C}_{n-|S|+1, m}$. Since $\Gamma$ is obtained by internal composition of $\Gamma_{S}$ and $\Gamma / \Gamma_{S}$, and the internal composition - has total degree -1 , then $\Gamma / \Gamma_{S}$ has degree 1 precisely when $\Gamma_{S}$ has degree 2 , and $\omega_{\Gamma_{S}} \wedge \omega_{\Gamma / \Gamma_{S}}$ is a top-dimensional differential form on $\bar{C}_{|S|} \times \bar{C}_{n-|S|+1, m}$ in this case.

Similarly, if $\left(S, S^{\prime}\right)$ are subsets of the internal and boundary vertices of $\Gamma$ respectively, with $2|S|+\left|S^{\prime}\right| \geq 2$, then we have a boundary component

$$
\iota_{S, S^{\prime}}: \bar{C}_{|S|,\left|S^{\prime}\right|} \times \bar{C}_{n-|S|, m-\left|S^{\prime}\right|+1} \hookrightarrow \bar{C}_{n, m}
$$

and, in case the subgraph $\Gamma_{S, S^{\prime}}$ is normal, we also have the differential form $\omega_{\Gamma_{S, S^{\prime}}} \wedge \omega_{\Gamma / \Gamma_{S, S^{\prime}}}$ on $\bar{C}_{|S|,\left|S^{\prime}\right|} \times \bar{C}_{n-|S|, m-\left|S^{\prime}\right|+1}$. Since the boundary composition 
of graphs has total degree zero, then $\Gamma_{S, S^{\prime}}$ has degree 1 precisely when also $\Gamma / \Gamma_{S, S^{\prime}}$ has degree one. In this case the differential form $\omega_{\Gamma_{S, S^{\prime}}} \wedge \omega_{\Gamma / \Gamma_{S, S^{\prime}}}$ on $\bar{C}_{|S|,\left|S^{\prime}\right|} \times \bar{C}_{n-|S|, m-\left|S^{\prime}\right|+1}$ is top-dimensional. One of the essential ingredients of Kontsevich's proof are the following compatibility equations: let $\Gamma$ be a degree 2 admissible graph, an let $S, S^{\prime}$ be such that $\operatorname{deg} \Gamma / \Gamma_{S}=\operatorname{deg} \Gamma / \Gamma_{S, S^{\prime}}=1$. Then

$$
\begin{gathered}
\iota_{S}^{*} \omega_{\Gamma}=\omega_{\Gamma_{S}} \wedge \omega_{\Gamma / \Gamma_{S}} \\
\iota_{S, S^{\prime}}^{*} \omega_{\Gamma}=\omega_{\Gamma_{S, S^{\prime}}} \wedge \omega_{\Gamma / \Gamma_{S, S^{\prime}}}
\end{gathered}
$$

The above compatibility equations tell us that $\omega$ can be seen as an EulerPoincaré map with respect to short exact sequences of admissible graphs [on03].

It remains to be seen what happens when one considers a full subgraph of $\Gamma$ which is not normal. Here is where the second main ingredient, the Kontsevich vanishing theorem, comes in: if $S$ or $\left(S, S^{\prime}\right)$ correspond to non-normal subgraphs, then

$$
\begin{gathered}
\int_{\bar{C}_{|S|} \times \bar{C}_{n-|S|+1, m}} \iota_{\bar{C}_{|S|,\left|S^{\prime}\right|} \times \bar{C}_{n-|S|, m-\left|S^{\prime}\right|+1}^{*} \omega_{\Gamma}=0} \iota_{S, S^{\prime}}^{*} \omega_{\Gamma}=0
\end{gathered}
$$

Indeed, non-normal subgraphs correspond to graphs with "bad edges" in Kontsevich's original notations, see Kon97, p.27. There is another vanishing theorem we will need in the next section: if $S$ is a subset of internal vertices of $\Gamma$ with $|S| \geq 3$ then the integral of $\omega_{\Gamma_{S}}$ on $\bar{C}_{|S|}$ vanishes. As a consequence,

$$
\int_{\bar{C}_{|S|} \times \bar{C}_{n-|S|+1, m}} \iota_{S}^{*} \omega_{\Gamma}=0 \quad \text { if }|S| \geq 3
$$

This means that, in order to have a non-vanishing integral we have to take $|S|=2$. But then, the requirement $\operatorname{deg} \Gamma_{S}=2$ forces $\Gamma_{S}$ to be an edge with two internal vertices; the configuration space $\bar{C}_{2}$ is diffeomorphic to $S^{1}$ and

$$
\int_{\bar{C}_{2}} \omega_{\Gamma_{S}}=\frac{1}{2 \pi} \int_{S^{1}} d \phi_{e}=1
$$

Summing up, from the compatibility and the vanishing equations above we have the following behavior for the boundary integrals of the $\omega_{\Gamma}$ 's: let $\Gamma$ be an admissible graph of total degree 2, with $n$ internal and $m$ boundary vertices. Then

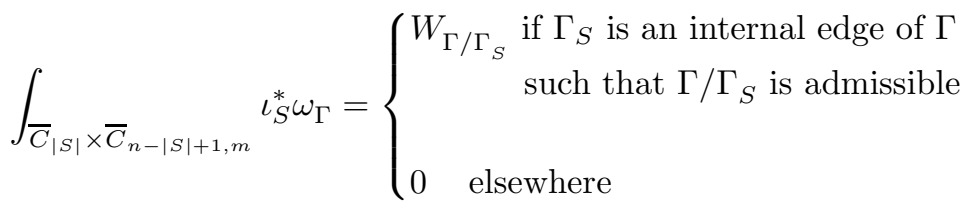


and

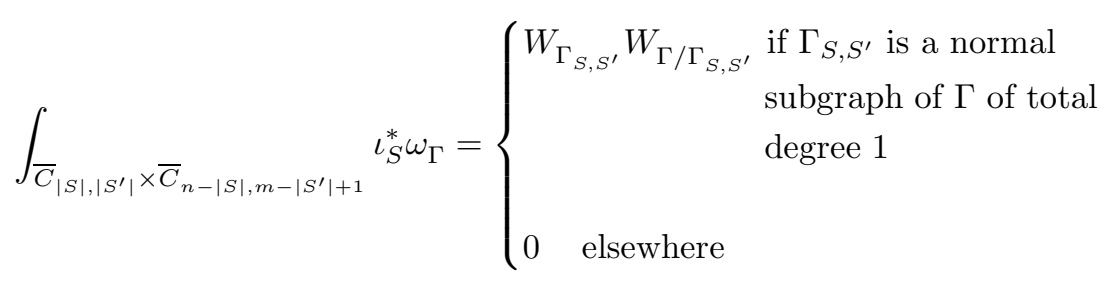

\section{A.3 Stokes' theorem and the cocycle equation}

We now turn back to Stokes' theorem:

$$
\int_{\partial \bar{C}_{n, m}} \omega_{\Gamma}=0
$$

for any admissible graph $\Gamma$ of total degree 2. According to the results of the previous subsection, this equation can be rewritten as

$$
\begin{aligned}
0 & =\sum_{S} \int_{\partial_{S} \bar{C}_{n, m}} \omega_{\Gamma}+\sum_{S, S^{\prime}} \int_{\partial_{S, S^{\prime}} \bar{C}_{n, m}} \omega_{\Gamma} \\
& =\sum_{e \in E_{\Gamma}} \pm W_{\Gamma / e}+\sum_{\substack{S, S^{\prime} \\
\Gamma_{S, S^{\prime}} \leqslant \Gamma}} \pm W_{\Gamma_{S, S^{\prime}}} W_{\Gamma / \Gamma_{S, S^{\prime}}}
\end{aligned}
$$

where the \pm signs depend on the orientation of the boundary components of $\bar{C}_{n, m}$. Due to the bijective correspondence between subsets of the vertices of $\Gamma$ and full subgraphs of $\Gamma$, we have

$$
\sum_{\substack{S, S^{\prime} \\ \Gamma_{S, S^{\prime}} \varangle \Gamma}} \pm W_{\Gamma_{S, S^{\prime}}} W_{\Gamma / \Gamma_{S, S^{\prime}}}=\sum_{\substack{\Phi \text { full, boundary } \\ \Phi \triangleleft \Gamma}} \pm W_{\Phi} W_{\Gamma / \Phi}
$$

Moreover, we have seen is Section [ 5 that a normal subgraph is necessarily full; so, summing up, we have found the equation

$$
\sum_{e \in E_{\Gamma}} \pm W_{\Gamma / e}+\sum_{\substack{\Phi \text { boundary } \\ \Phi \leqslant \Gamma}} \pm W_{\Phi} W_{\Gamma / \Phi}=0,
$$

that is,

$$
W\left(d_{2}^{*} \Gamma\right)+\frac{1}{2} W^{\otimes 2}(\delta \Gamma)=0, \quad \forall \Gamma \in \mathcal{G}^{2},
$$

i.e. the Kontsevich integral $W$ is a cocycle.

Remark A.2. One explicitly computes $W_{b_{0,2}}=1$ and $W_{b_{1, m}}=1 / m$ !. Thus the element $\sum_{\Gamma \in \mathcal{G}^{1}} W_{\Gamma} \Gamma$ is a solution of the Maurer-Cartan equation on graphs fulfilling the conditions from Proposition [7.1 and Remark 7.2 In particular Kontsevich's weights define a star-product deformation quantization of Poisson structures. 


\section{References}

[AIS05] Fusun Akman, Lucian M. Ionescu and Papa A. Sissokho. On deformation theory and graph homology. math.QA/0507077

[ABM99] Didier Arnal, Nabiha Ben Amar, Mohsen Masmoudi. Cohomology of good graphs and Kontsevich linear star products, Lett. Math. Phys. 48 (1999), no. 4, 291-306.

[AM02] Didier Arnal and Mohsen Masmoudi. Cohomologie de Hochschild des graphes de Kontsevich. Bull. Soc. Math. France, 130(1):49-69, 2002.

[AMM02] Didier Arnal, Dominique Manchon and Mohsen Masmoudi. Choix des signes pour la formalité de M. Kontsevich. Pacific J. Math., 203(1):23-66, 2002.

[Arn04] Didier Arnal. Kontsevich formality and cohomologies for graphs. Lett. Math. Phys., 69:205-222, 2004.

[Cat04] Alberto S. Cattaneo. Formality and star products, Lecture notes by Davide Indelicato. math.QA/0403135.

[CDF03] Alberto S. Cattaneo, Benoit Dherin, and Giovanni Felder. Formal symplectic groupoid. Comm. Math. Phys., 253(3):645-674, 2005. math.SG/0312380

[CF99] Alberto S. Cattaneo and Giovanni Felder. A path integral approach to the Kontsevich quantization formula. Comm. Math. Phys., 212(3):591-611, 2000. math.QA/9902090.

[CK98] Alain Connes and Dirk Kreimer. Hopf algebras, renormalization and noncommutative geometry. Comm. Math. Phys., 199(1):203-242, 1998. hep-th/9808042.

[Dit99] Giuseppe Dito. Kontsevich star products on the dual of a Lie algebra. Lett. Math. Phys. 48 (1999), 307-322. math.QA/9905080

[DS02] Giuseppe Dito and Daniel Sternheimer. Deformation quantization: genesis, developments and metamorphoses. In Deformation quantization (Strasbourg, 2001), volume 1 of IRMA Lect. Math. Theor. Phys., pages 9-54. de Gruyter, Berlin, 2002. math.QA/0201168

[GaH03] Angela Gamella and Gilles Halbout. $G_{\infty}$-formality theorem in terms of graphs and associated Chevalley-EilenbergHarrison cohomology. preprint IRMA 2003-05-21 $\mathrm{n}^{\circ}$ 2003-015, http://www-irma.u-strasbg.fr/irma/publications/2003/03015.shtml

[GiH03] Grégory Ginot and Gilles Halbout. Lifts of $C_{\infty}$ and $L_{\infty^{-}}$ morphisms to $G_{\infty}$-morphisms. Proc. Amer. math. Soc., to appear. math.QA/0304004 
[Ion03] Lucian M. Ionescu. Perturbative quantum field theory and configuration space integrals. hep-th/0307062.

[Ion04a] Lucian M. Ionescu. A combinatorial approach to coefficients in deformation quantization. math.QA/0404389

[Ion04b] Lucian M. Ionescu. Cohomology of Feynman graphs and perturbative quantum field theory. In O. Kovras, editor, Focus on Quantum Field Theory, volume 1. Nova Science Publishers, Inc., 2004. math.QA/0506142

[Ion04c] Lucian M. Ionescu. Perturbative Quantum Field Theory and $L_{\infty^{-}}$ algebras. In J. Bryden, editor, Advances in Topological Field Theory, Proceedings of the NATO ARW on New Techniques in Topological Quantum Field Theory, pages 243-252. Kluwer Academic Publishers, 2004 .

[IS05] Lucian M. Ionescu and Papa A. Sissokho. A canonical semi-classical star-product. math.QA/0507053

[Jon02] John D. S. Jones, Lectures on operads. Contemporary Methematics 315, p.89-130, 2002.

[Kat98] Vinay Kathotia. Kontsevich's universal formula for deformation quantization and the Campbell-Baker-Hausdorff formula. Internat. J. Math., 11(4):523-551, 2000. math.QA/9811174

[Kel] Bernhard Keller. Notes for an Introduction to Kontsevich's quantization theorem.

http://www.math.jussieu.fr/ keller/publ/emalca.pdf

[Kon93] Maxim Kontsevich. Formal (non)commutative symplectic geometry. In The Gel'fand Mathematical Seminars, 1990-1992, pages 173-187. Birkhäuser Boston, Boston, MA, 1993.

[Kon92] Maxim Kontsevich. Feynman diagrams and low-dimensional topology. In First European Congress of Mathematics, Vol. II (Paris, 1992), volume 120 of Progr. Math., pages 97-121. Birkhäuser, Basel, 1994.

[Kon96] Maxim Kontsevich. Formality conjecture. In Deformation theory and symplectic geometry (Ascona, 1996), volume 20 of Math. Phys. Stud., pages 139-156. Kluwer Acad. Publ., Dordrecht, 1997.

[Kon99] Maxim Kontsevich. Operads and motives in deformation quantization. Lett. Math. Phys., 48(1):35-72, 1999. math.QA/9904055.

[Kon97] Maxim Kontsevich. Deformation quantization of Poisson manifolds. Lett. Math. Phys., 66(3):157-216, 2003. q-alg/9709040. 
[KS00] Maxim Kontsevich and Yan Soibelman. Homological mirror symmetry and torus fibrations. In Symplectic geometry and mirror symmetry (Seoul, 2000), pages 203-263. World Sci. Publishing, River Edge, NJ, 2001. math.SG/0011041.

[Mar99] Martin Markl. Homotopy algebras are homotopy algebras. Forum Math. 16(1), 129-160 (2004). math.AT/9907138

[Moc02] Takuro Mochizuki. On the morphism of Duflo-Kirillov type. J. Geom. Phys., 41(1-2):73-113, 2002.

[Pol02] Michael Polyak. Quantization of linear Poisson structures and degrees of maps. Lett. Math. Phys., 66(1-2):15-35, 2003. math.GT/0210107

[Qui69] Daniel Quillen. Rational homotopy theory. Ann. of Math., 90, p. 205-295, 1969.

[Vor] Alexander A. Voronov. Lecture notes on Operads and Quantum Field Theory. Lecture 9: The $A_{\infty}$-operad and $A_{\infty}$-algebras. http://www. math.umn.edu/ voronov/8390 